\title{
EXAMPLES OF FACTORS WHICH HAVE NO CARTAN SUBALGEBRAS
}

\begin{abstract}
YUSUKE ISONO
Abstract. We consider some conditions similar to Ozawa's condition (AO) and prove that if a non-injective factor satisfies such a condition and has the $\mathrm{W}^{*} \mathrm{CBAP}$, then it has no Cartan subalgebras. As a corollary, we prove that $\mathrm{II}_{1}$ factors of universal orthogonal and unitary discrete quantum groups have no Cartan subalgebras. We also prove that continuous cores of type $\mathrm{III}_{1}$ factors with such a condition are semisolid as a $\mathrm{II}_{\infty}$ factor.
\end{abstract}

\section{INTRODUCTION}

In the von Neumann algebra theory, the Cartan subalgebras give us much important information and fascinating examples. In fact, Cartan subalgebras always come from some orbit equivalence classes in the following sense: for a given separable factor $M$ and its Cartan subalgebra $A \subset M$, there exists the unique orbit equivalence class $\mathcal{R}$ (and the cocycle $\sigma$ ) on a standard space $X$ such that $\left(L^{\infty}(X) \subset L(\mathcal{R}, \sigma)\right) \simeq(A \subset M)$ [12]. This correspondence sometimes enables us to make use of the ergodic theory to analyze such a class of factors. This is one of the main reasons why Cartan subalgebras have been studied for a long time.

For example, Sorin Popa gave the first examples of $\mathrm{II}_{1}$ factors whose fundamental groups are trivial [26. In the proof, he identified fundamental groups of these factors as that of orbit equivalence classes by some deformation/intertwining arguments between two Cartan subalgebras. Hence he essentially investigated their Cartan subalgebras. This is the first result of the rigidity theory of $\mathrm{II}_{1}$ factors.

From this pioneering work, there have been many remarkable works: realization of many outer automorphism groups and fundamental groups; new examples of prime factors; uniqueness and non-existence of Cartan subalgebras; $\mathrm{W}^{*}$ superrigidity and so on. In this paper, we concentrate our attention on a negative type result, that is, non-existence of Cartan subalgebras.

Here we recall the definition of Cartan subalgebras. Let $M$ be a von Neumann algebra and $A$ an abelian subalgebra of $M$. We say $A$ is a Cartan subalgebra of $M$ if it satisfies the following conditions:

- there exists a faithful normal conditional expectation from $M$ onto $A$;

- $A$ is maximal abelian in $M$, that is, $A^{\prime} \cap M=A$;

- the normalizer group $\mathcal{N}_{M}(A)$ generates $M$, that is, $\mathcal{N}_{M}(A)^{\prime \prime}=M$.

Here the normalizer group is defined as $\mathcal{N}_{M}(A):=\left\{u \in \mathcal{U}(M) \mid u A u^{*}=A\right\}$. We note that Cartan subalgebras of $M$ are diffuse (i.e. which have no minimal projections) if so is $M$.

Received by the editors October 10, 2012 and, in revised form, November 23, 2012, March 13, 2013, June 23, 2013, and August 16, 2013.

2010 Mathematics Subject Classification. Primary 20G42, 46L10. 
Historically, examples of von Neumann algebras which have no Cartan subalgebras were first discovered by Voiculescu [36. He showed that the free group factors $L \mathbb{F}_{n}(n \geq 2)$ have no Cartan subalgebras and his method relied on the free probability theory. Jung generalized this result to some free product von Neumann algebras [19]. Shlyakhtenko proved that free Araki-Woods factors of type $\mathrm{III}_{\lambda}$ have no Cartan subalgebras 29 .

In the rigidity theory, Ozawa and Popa gave the first examples 24. They proved that the free group factors are such examples, and they actually proved that these factors are strongly solid. Here we recall that a finite von Neumann algebra $M$ is strongly solid if for any diffuse injective subalgebra $A \subset M$, the normalizer $\mathcal{N}_{M}(A)$ generates an injective von Neumann algebra; that is, $\mathcal{N}_{M}(A)^{\prime \prime}$ is injective. It is easy to see that if a finite von Neumann algebra $M$ is strongly solid, then any noninjective diffuse von Neumann subalgebra of $M$ has no Cartan subalgebras. Hence their result is stronger than that of Voiculescu.

After the work of Ozawa and Popa, there have been many non-existence results, and in the present paper we follow [27, in which Popa and Vaes proved remarkable uniqueness and non-existence results of Cartan subalgebras. In the same paper they gave a new proof of the fact that factors of weakly amenable and bi-exact groups are strongly solid (this was first proved by Chifan, Sinclair, and Udrea [9, Corollary 0.2] with an equivalent notion of bi-exactness called array; see [8] and [9. Proposition 2.1]). We refer to this new proof. In fact, we will prove the same statement for more general von Neumann algebras which are not necessarily group von Neumann algebras.

For this purpose, we need notions of general von Neumann algebras which correspond to weak amenability and bi-exactness. It is known that weak amenability has such a notion called the $\mathrm{W}^{*} \mathrm{CBAP}$ (see Subsection 2.3), but bi-exactness does not. Ozawa's condition (AO) is a candidate, but this is not enough for us. We will investigate it in Section 3] See [28, 14], and [18] for other notions similar to condition $(\mathrm{AO})$.

After this consideration, we prove the following main theorems.

Theorem A. Let $M$ be a $\mathrm{II}_{1}$ factor with separable predual. If $M$ satisfies condition $(\mathrm{AO})^{+}$(see Definition 3.1.1) and has the $W^{*} C B A P$, then $M$ is strongly solid.

Theorem B. Let $M$ be a non-injective type III factor with separable predual and $\phi$ a faithful normal state on $M$. If $(M, \phi)$ satisfies condition $(\mathrm{AOC})^{+}$(see Definition 3.2.1) and has the $W^{*} C B A P$, then $M$ has no $\phi$-Cartan subalgebras.

Here $\phi$-Cartan subalgebra means a Cartan subalgebra which has a $\phi$-preserving faithful normal conditional expectation $E$, that is, $\phi=\phi \circ E$.

To prove Theorem $\mathrm{A}$, we need only slight modifications of the proof of (a special case of) [27, Theorem 3.1]. Theorem B can be proved by seeing its continuous core, and this idea comes from [16] and [17. Since condition $(A O C)^{+}$is similar to condition ( $\mathrm{AO}$ ) with respect to the continuous core, we naturally deduce the following primeness result. In the theorem below, Tr means the canonical semifinite trace on the continuous core.

Theorem C. Let $M$ be a von Neumann algebra with separable predual and $\phi$ a faithful normal state on $M$. Let $\mathcal{M}$ be its continuous core with respect to $\phi$ and $p$ a projection in $\mathcal{M}$ such that $\operatorname{Tr}(p)<\infty$. If $(M, \phi)$ satisfies condition $(\mathrm{AOC})^{+}$, then 
$p \mathcal{M} p$ is semisolid. In particular, $\mathcal{M}$ is a semisolid type $\mathrm{II}_{\infty}$ factor if $M$ is a type $\mathrm{III}_{1}$ factor.

Here we recall that a finite von Neumann algebra $M$ is semisolid (respectively, solid) if for any type II (respectively, diffuse) subalgebra $N \subset M$, the relative commutant $N^{\prime} \cap M$ is injective. For a semifinite von Neumann algebra $M$, semisolidity (and solidity) is defined as that of $p M p$ for all finite projections $p \in M$. We also recall that $M$ is prime if for any tensor decomposition $M=M_{1} \otimes M_{2}$, one of $M_{i}$ $(i=1,2)$ is of type I. It is not difficult to see that semisolidity implies primeness for non-injective semifinite factors. Hence the conclusion of the theorem above implies primeness.

The aim of our generalization is, of course, to find new examples. Factors of universal discrete quantum groups $A_{o}(F)$ and $A_{u}(F)$ (see Subsection 2.4) are our main targets. On the one hand, it is known that they satisfy condition (AO) 34, [33, and we will observe that they in fact satisfy slightly stronger conditions. See Section 3 for the details. On the other hand, weak amenability of them was shown very recently [13] but only for the case where they are monoidally equivalent to $A_{o}\left(1_{n}\right)$ or $A_{u}\left(1_{n}\right)$. Thus combined with the main theorems, we have the following corollary.

Corollary. Let $\mathbb{G}$ be a universal discrete quantum group $A_{o}(F)$ or $A_{u}(F)$ for $F \in$ $\operatorname{GL}(n, \mathbb{C})(n \geq 3)$. Denote the Haar state by $h$.

(1) If $F=1_{n}$, then $L^{\infty}(\mathbb{G})$ is strongly solid. In particular, $L^{\infty}(\mathbb{G})$ has no Cartan subalgebras.

(2) If $L^{\infty}(\mathbb{G})$ is non-injective and has the $W^{*} C B A P$, then it has no h-Cartan subalgebras.

(3) If $L^{\infty}(\mathbb{G})$ is a non-injective type $\mathrm{III}_{1}$ factor, then the continuous core $L^{\infty}(\mathbb{G}) \rtimes_{\sigma^{h}} \mathbb{R}$ is a semisolid, in particular prime, $\mathrm{II}_{\infty}$ factor.

We will observe in Subsection 5.2 that the continuous core of $L^{\infty}\left(A_{o}(F)\right)$ is semisolid but never solid for some concrete matrix $F$.

Theorem $\mathrm{B}$ works for the case that $F$ is not an identity matrix, but we do not know whether $L^{\infty}(\mathbb{G})$ has the $\mathrm{W}^{*} \mathrm{CBAP}$ or not for a general matrix $F$. If one obtains this property, Theorem B is applicable for every non-injective von Neumann algebra of $A_{o}(F)$ and $A_{u}(F)$, and hence one has non-existence results for them. We state this problem as follows.

Problem. When do von Neumann algebras of universal discrete quantum groups $A_{o}(F)$ and $A_{u}(F)$ have the $\mathrm{W}^{*} \mathrm{CBAP}$ ?

\section{Preliminaries}

2.1. Tomita-Takesaki theory. For Tomita-Takesaki theory, we refer the reader to 30 .

Let $M$ be a von Neumann algebra and $\phi$ a faithful normal state on $M$. We first consider the following anti-linear map:

$$
S: M \Omega \rightarrow M \Omega \subset L^{2}(M, \phi) ; a \Omega \mapsto a^{*} \Omega,
$$

where $\Omega$ is the canonical cyclic separating vector associated with $\phi$. This map is closable on $L^{2}(M, \phi)$, and we write as $S=J \Delta^{1 / 2}$ the polar decomposition of $S$. 
We call $\Delta$ the modular operator and $J$ the modular conjugation. The following fundamental relations are important:

$$
J M J=M^{\prime}, \quad \Delta^{i t} M \Delta^{-i t}=M \quad(t \in \mathbb{R}) .
$$

In the paper, we frequently identify $J M J$ as the opposite algebra $M^{\text {op }}$ with the obvious correspondence. The GNS-representation on the Hilbert space $L^{2}(M, \phi)$ (with a faithful normal state $\phi$ ) is called a standard representation (see [30, Definition IX.1.14] for the intrinsic definition).

From the relation above, $\sigma_{t}^{\phi}(a):=\Delta^{i t} a \Delta^{-i t}(a \in M, t \in \mathbb{R})$ defines a one parameter automorphism group on $M$, which is called the modular automorphism group on $M$ associated with $\phi$. The continuous core of $M$ is defined as the crossed product von Neumann algebra $\widetilde{M}:=M \rtimes_{\sigma^{\phi}} \mathbb{R}$ and it does not depend on the choice of $\phi$. We can then construct a semifinite weight $\tilde{\phi}$ on the core called the dual weight of $\phi$ [30, Definition X.1.16], which of course depends on $\phi$. The dual weights are always faithful and their modular action becomes inner (more precisely $\sigma_{t}^{\tilde{\phi}}=1 \otimes \lambda_{t}$ ) so that $\widetilde{M}$ is always semifinite. A canonical semifinite trace on $\widetilde{M}$ is given by $\operatorname{Tr}:=\tilde{\phi}(h \cdot)$, where $h$ is the self-adjoint map satisfying $h^{i t}=1 \otimes \lambda_{t}(t \in \mathbb{R})$. We say a type III factor $M$ is of type $\mathrm{III}_{1}$ if the continuous core is a factor of type $\mathrm{II}_{\infty}$ (see [30, Definition XII.1.3] for definitions of type $\mathrm{III}_{\lambda}$ ).

The associated representations

$$
\begin{aligned}
& \pi: M \rightarrow \mathbb{B}\left(L^{2}(M) \otimes L^{2}(\mathbb{R})\right) ; x \mapsto \int_{\mathbb{R}} \sigma_{-t}^{\phi}(x) \otimes e_{t} \cdot d t, \\
& u: \quad \mathbb{R} \rightarrow \mathcal{U}\left(L^{2}(M) \otimes L^{2}(\mathbb{R})\right) ; t \mapsto 1 \otimes \lambda_{t},
\end{aligned}
$$

where $\left(\int_{\mathbb{R}} \sigma_{-t}^{\phi}(x) \otimes e_{t} \cdot d t \xi\right)(s):=\sigma_{-s}^{\phi}(x) \xi(s)$ and $\left(\left(1 \otimes \lambda_{t}\right) \xi\right)(s):=\xi(-t+s)$ for any $\xi \in L^{2}(M) \otimes L^{2}(\mathbb{R})$, give a standard representation of $M \rtimes_{\sigma^{\phi}} \mathbb{R}$ on $L^{2}(M) \otimes L^{2}(\mathbb{R})$ with respect to the dual weight $\tilde{\phi}$.

A conditional expectation from $\widetilde{M}$ onto $L \mathbb{R}$ is defined by $E_{L \mathbb{R}}\left(x \lambda_{t}\right):=\phi(x) \lambda_{t}$ $(x \in M, t \in \mathbb{R})$. Then $E_{L \mathbb{R}}$ is $\tilde{\phi}$-preserving and Tr-preserving. The modular conjugation $\tilde{J}$ on $\widetilde{M}$ is given by

$$
(\tilde{J} \xi)(t):=\Delta^{-i t} J \xi(-t) \quad\left(t \in \mathbb{R}, \xi \in L^{2}(M) \otimes L^{2}(\mathbb{R})\right)
$$

[30, Lemma X.1.13], and one can easily verify that

$$
\tilde{J} \pi(x) \tilde{J}=J x J \otimes 1 \quad(x \in M), \quad \tilde{J}\left(1 \otimes \lambda_{t}\right) \tilde{J}=\Delta^{i t} \otimes \rho_{t} \quad(t \in \mathbb{R}),
$$

where $\rho_{t}$ is the right translation defined by $\left(\rho_{t} \eta\right)(s):=\eta(s+t)\left(\eta \in L^{2}(\mathbb{R})\right)$. Hence we have

$$
\begin{aligned}
\left(M \rtimes_{\sigma^{\phi}} \mathbb{R}\right)^{\prime}=\tilde{J}\left(M \rtimes_{\sigma^{\phi}} \mathbb{R}\right) \tilde{J} & =W^{*}\left\{J x J \otimes 1(x \in M), \Delta^{i t} \otimes \rho_{t}(t \in \mathbb{R})\right\} \\
& =W^{*}\left\{M^{\prime} \otimes 1, \Delta^{i t} \otimes \rho_{t}(t \in \mathbb{R})\right\} .
\end{aligned}
$$

Next, we investigate how Cartan subalgebras of $M$ behave in the continuous core of $M$. Let $M$ be a general von Neumann algebra, $A \subset M$ a Cartan subalgebra of $M$, and $E_{A}$ an associated conditional expectation. Take a faithful normal state $\phi$ on $A$ and extend it on $M$ via $E_{A}$ (still denote it by $\phi$ ). Then by the proof of Takesaki's conditional expectation theorem [30, Theorem IX.4.2], the restriction of $\sigma_{t}^{\phi}$ on $A$ coincides with the modular automorphism group on $A$ associated with $\phi$. This implies $\sigma_{t}^{\phi}(A)=A$ so that we have a natural inclusion $A \rtimes_{\sigma^{\phi}} \mathbb{R} \subset M \rtimes_{\sigma^{\phi}} \mathbb{R}$. Since $A$ is abelian (and so $\phi$ is tracial), $\sigma_{t}^{\phi}=\operatorname{id}_{A}$ on $A$ and hence we have $A \rtimes_{\sigma^{\phi}} \mathbb{R}=A \otimes L \mathbb{R}$. 
Then it is known that for any Tr-finite projection $p \in L \mathbb{R}$, the reduced subalgebra $A \otimes p L \mathbb{R} p$ is a Cartan subalgebra of a finite von Neumann algebra $p\left(M \rtimes_{\sigma^{\phi}} \mathbb{R}\right) p$ (see e.g. [16, Propositions 2.6 and 2.7] and [11, Lemma 2.2]).

2.2. Popa's intertwining techniques. As explained in the Introduction, Sorin Popa introduced a useful tool which gives a good sufficient condition for unitary conjugacy of Cartan subalgebras. Here we recall only the precise statement which we will need later. See [4, Theorem F.12] for another proof.

Theorem 2.2.1 (26, 25]). Let $M$ be a finite von Neumann algebra with separable predual, $\tau$ a faithful normal trace on $M$, and $A, B \subset M$ (possibly non-unital) von Neumann subalgebras. We denote by $E_{B}$ the unique $\tau_{B}$-preserving conditional expectation from $1_{B} M 1_{B}$ onto $B$, where $\tau_{B}:=\tau\left(1_{B} \cdot 1_{B}\right) / \tau\left(1_{B}\right)$. Then the following conditions are equivalent.

(1) There exist no sequences $\left(w_{n}\right)_{n}$ of unitaries in $A$ such that $\lim _{n}\left\|E_{B}\left(b^{*} w_{n} a\right)\right\|_{2, \tau_{B}}=0$ for any $a, b \in 1_{A} M 1_{B}$.

(2) There exists a non-zero $A$-B-submodule $H$ of $1_{A} L^{2}(M, \tau) 1_{B}$ such that $\operatorname{dim}_{\left(B, \tau_{B}\right)} H<\infty$.

(3) There exist non-zero projections $e \in A$ and $f \in B$, a unital normal $*$ homomorphism $\theta: e A e \rightarrow f B f$, and a partial isometry $v \in M$ such that

- $v v^{*} \leq e$ and $v^{*} v \leq f$,

- $v \theta(x)=x v$ for any $x \in e A e$.

We write $A \preceq_{M} B$ if one of these conditions holds.

In the statement of $(2)$ above, we do not need to take $L^{2}(M, \tau)$. In fact, we can choose any standard representation of $M$, since all standard representations are canonically isomorphic with each other including the left and right actions of $M$ (and hence those of $A$ and $B$ ).

2.3. Weak amenability and $\mathbf{W}^{*} \mathbf{C B A P}$. Weak amenability is an approximation property for discrete groups (more generally, locally compact groups) weaker than amenability, and the $\mathrm{W}^{*} \mathrm{CBAP}$ is a corresponding notion for von Neumann algebras.

To introduce these notions, we first recall the definition of a Herz-Shur multiplier. Let $\Gamma$ be a discrete group and $\phi$ a map from $\Gamma$ to $\mathbb{C}$. Consider a linear map

$$
m_{\phi}: \mathbb{C}[\Gamma] \rightarrow \mathbb{C}[\Gamma] ; \sum_{s \in \Gamma} a_{s} \cdot s \mapsto \sum_{s \in \Gamma} \phi(s) a_{s} \cdot s .
$$

Then regarding $\mathbb{C}[\Gamma] \subset C_{\lambda}^{*}(\Gamma)$, we define the Herz-Shur norm of $\phi$ as $\|\phi\|_{\text {c.b. }}:=$ $\left\|m_{\phi}\right\|_{\text {c.b. }}$ (possibly infinite). We say $\phi$ (or $\left.m_{\phi}\right)$ is a Herz-Shur multiplier if $\|\phi\|_{\text {c.b. }}$ is finite.

Then recall that a discrete group $\Gamma$ is weakly amenable if there exists a net $\left(\phi_{i}\right)_{i}$ of finitely supported Herz-Shur multipliers satisfying $\lim \sup _{i}\left\|\phi_{i}\right\|_{\text {c.b. }}<\infty$ and $\phi_{i}(g) \rightarrow 1$ as $i \rightarrow \infty$ for any $g \in \Gamma$. We also recall that a von Neumann algebra $M$ has the weak ${ }^{*}$ completely bounded approximation property (or $W^{*} C B A P$, in short) if there exists a net $\left(\psi_{i}\right)_{i}$ of normal c.b. maps on $M$ with finite rank such that $\lim \sup _{i}\left\|\psi_{i}\right\|_{\text {c.b. }}<\infty$ and $\psi_{i}$ converges to $\operatorname{id}_{M}$ in the point $\sigma$-weak topology.

Then optimal constants

$\Lambda_{\text {c.b. }}(\Gamma):=\inf \left\{\lim \sup \left\|\phi_{i}\right\|_{\text {c.b. }} \mid\left(\phi_{i}\right)\right.$ satisfies the above condition $\}$,

$\Lambda_{\text {c.b. }}(M):=\inf \left\{\limsup \left\|\psi_{i}\right\|_{\text {c.b. }} \mid\left(\psi_{i}\right)\right.$ satisfies the above condition $\}$ 
are invariants of $\Gamma$ and $M$ respectively, both of which are called the CowlingHaagerup constant. It is known that $\Lambda_{\text {c.b. }}(\Gamma)=\Lambda_{\text {c.b. }}(L \Gamma)$ (see for example [4, Section 12.3]). Recently combined with an approximation property result of Brannan [5], Freslon proved that $\Lambda_{\text {c.b. }}\left(L^{\infty}(\mathbb{G})\right)=1$, where $\mathbb{G}$ is monoidally equivalent to $A_{o}\left(1_{n}\right)$ or $A_{u}\left(1_{n}\right)$ [13. However the general case is still open.

We will use these properties in two ways: one is Theorem 4.1.1 to get weakly compact actions; the other is as follows with local reflexivity. Recall a $C^{*}$-algebra $A$ is locally reflexive if for any finite dimensional subspace $E \subset A^{* *}$ there exists a net $\left(\mu_{j}\right)_{j}$ of c.c. maps from $E$ to A such that $\left(\mu_{j}\right)_{j}$ converges to $\mathrm{id}_{E}$ in the point $\sigma$-weak topology.

Lemma 2.3.1. Let $M$ be a von Neumann algebra and $A \subset M$ a $\sigma$-weakly dense $C^{*}$ subalgebra. Let $\left(\phi_{i}\right)_{i}$ be a net of normal c.b. maps on $M$ with finite rank such that $\lim \sup _{i}\left\|\phi_{i}\right\|_{\text {c.b. }}=: k<\infty$ and $\phi_{i}$ converges to $\operatorname{id}_{M}$ in the point $\sigma$-weak topology. Assume $A$ is locally reflexive. Then we can find a net $\left(\psi_{j}\right)_{j}$ of normal c.b. maps from $M$ into $A$ with finite rank satisfying the same conditions as $\left(\phi_{i}\right)_{i}$.

Proof. Let $z \in A^{* *}$ be the central projection satisfying $M \simeq z A^{* *}$. Put $E_{i}:=\phi_{i}(M)$ and regard it as a subset of $A^{* *}$ via $E_{i} \subset M \simeq z A^{* *}$. Then, by local reflexivity of $A$, we can find a net $\left(\mu_{j}^{i}\right)_{j}$ of c.c. maps from $E_{i}$ into $A$ such that $\mu_{j}^{i}$ converges to $\operatorname{id}_{E_{i}}$ in the point $\sigma$-weak topology. Now, putting $\tilde{\mu}_{j}^{i}(a):=z \mu_{j}^{i}(a)$, we have a net $\left(\tilde{\mu}_{j}^{i} \circ \phi_{i}\right)_{i, j}$ of c.b. maps from $M$ into $z A$, and this makes our desired net by using the identification $(A \subset M) \simeq\left(z A \subset z A^{* *}\right)$.

2.4. Universal discrete quantum groups. In the paper, we use the quantum group theory only for Propositions 3.1.2 and 3.2.3. We accept all the basics of compact and discrete quantum groups and we refer the reader to [37] and [20] for the details. Our notations are very similar to those of 34 .

Let $C(\mathbb{G})$ be a compact quantum group. We denote by $\Phi$ the comultiplication, by $h$ the Haar state, and by $L^{2}(\mathbb{G})$ the GNS-representation of $h$. Then the Hilbert space $L^{2}(\mathbb{G})$ can be decomposed as follows:

$$
L^{2}(\mathbb{G})=\sum_{x \in \operatorname{Irred}(\mathbb{G})} \bigoplus\left(H_{x} \otimes H_{\bar{x}}\right),
$$

where $\operatorname{Irred}(\mathbb{G})$ is the set of equivalent classes of all irreducible unitary representations of $\mathbb{G}$ and $\bar{x}$ is the contragredient of $x$. Let $t_{x}$ be the unique unit vector (up to multiplication by $\mathbb{T})$ in $H_{x} \otimes H_{\bar{x}}$ such that it is $\left(U^{x} \otimes U^{\bar{x}}\right)$-invariant, where $U^{x}$ is the unitary element corresponding to $x$. Identify $t_{x}$ as an anti-linear map from $H_{\bar{x}}$ to $H_{x}$ with the Hilbert-Schmidt correspondence. Then we have two representations,

$$
\begin{aligned}
& \rho: C(\mathbb{G}) \rightarrow \mathbb{B}\left(L^{2}(\mathbb{G})\right) ; \rho\left(\omega_{\eta, \xi} \otimes \iota\left(U^{x}\right)\right) \Omega=\xi \otimes t^{\bar{x}} \eta \in H_{x} \otimes H_{\bar{x}}, \\
& \lambda: C(\mathbb{G}) \rightarrow \mathbb{B}\left(L^{2}(\mathbb{G})\right) ; \lambda\left(\omega_{\eta, \xi} \otimes \iota\left(U^{x}\right)\right) \Omega=t^{\bar{x}} \eta \otimes \xi \in H_{\bar{x}} \otimes H_{x},
\end{aligned}
$$

for all $x \in \operatorname{Irred}(\mathbb{G})$ and $\xi, \eta \in H_{x}$. Here $\Omega$ is the canonical cyclic vector. We note that these representations are unitarily equivalent to the GNS-representation for the Haar state $h$. Define the dual discrete quantum group as

$$
\begin{aligned}
c_{0}(\hat{\mathbb{G}}) & :=\bigoplus_{x \in \operatorname{Irred}(\mathbb{G})} \mathbb{B}\left(H_{x}\right), \\
\ell^{\infty}(\hat{\mathbb{G}}) & :=\prod_{x \in \operatorname{Irred}(\mathbb{G})} \mathbb{B}\left(H_{x}\right),
\end{aligned}
$$


and define two representations of them on the same Hilbert space $L^{2}(\mathbb{G})$ by

$$
\begin{aligned}
& \hat{\lambda}: \ell^{\infty}(\hat{\mathbb{G}}) \rightarrow \prod_{x \in \operatorname{Irred}(\mathbb{G})} \mathbb{B}\left(H_{x}\right) \otimes \mathbb{C} \subset \mathbb{B}\left(L^{2}(\mathbb{G})\right), \\
& \hat{\rho}: \ell^{\infty}(\hat{\mathbb{G}}) \rightarrow \prod_{\bar{x} \in \operatorname{Irred}(\mathbb{G})} \mathbb{C} \otimes \mathbb{B}\left(H_{x}\right) \subset \mathbb{B}\left(L^{2}(\mathbb{G})\right) .
\end{aligned}
$$

All dual objects are written with a hat (e.g. $\hat{\Phi}, \hat{h})$. We have a natural unitary

$$
\mathbb{V}=\bigoplus_{x \in \operatorname{Irred} \mathbb{G}} U^{x}
$$

From now on, we assume that the Haar state $h$ is faithful on $C(\mathbb{G})$ and recall modular objects of them. We use similar notation to [31], which has a good survey of the modular theory on compact quantum groups. Let $A(\mathbb{G})$ be the dense Hopf *-algebra of $C(\mathbb{G}), \kappa$ the antipode, and $\epsilon$ the counit of $C(\mathbb{G})$. Let $\left\{f_{z}\right\}_{z}(z \in \mathbb{C})$ be the Woronowicz characters on $C(\mathbb{G})$, that is, a family of homomorphisms from $A(\mathbb{G})$ to $\mathbb{C}$ satisfying conditions in $\left[37\right.$, Theorem 1.4]. Put $F_{x}:=\left(\iota \otimes f_{1}\right)\left(U^{x}\right)$ for $x \in \operatorname{Irred}(\mathbb{G})$. Then we have the following useful relations to the modular group associated with the Haar state $h$ :

$$
\begin{aligned}
& \text { - }\left(\iota \otimes \sigma_{t}^{h}\right)\left(U^{x}\right)=\left(F_{x}^{i t} \otimes 1\right) U^{x}\left(F_{x}^{i t} \otimes 1\right) \quad(t \in \mathbb{R}, x \in \operatorname{Irred}(\mathbb{G})), \\
& \text { - } \Delta^{i t}=\sum_{x \in \operatorname{Irred}(\mathbb{G})} \bigoplus\left(F_{x}^{i t} \otimes F_{\bar{x}}^{-i t}\right) \quad \text { on } L^{2}(\mathbb{G})=\sum_{x \in \operatorname{Irred}(\mathbb{G})} \bigoplus\left(H_{x} \otimes H_{\bar{x}}\right) .
\end{aligned}
$$

We denote the scaling automorphism group by $\tau_{t}$ and the unitary antipode by $R$. Define a conjugate unitary $\hat{J}$ on $L^{2}(\mathbb{G})$ by $\hat{J} x \hat{1}:=R\left(x^{*}\right) \hat{1}$ for $x \in C(\mathbb{G})$ and put $U:=J \hat{J}=\hat{J} J$. Then we can identify all compact quantum group $C^{*}$-algebras as these opposite algebras.

Next we recall universal discrete quantum groups introduced in 35 which are our main objects. Let $F$ be an element in $\operatorname{GL}(n, \mathbb{C})(n \geq 2)$. Then the $C^{*}$-algebra $C\left(A_{u}(F)\right)$ (respectively, $C\left(A_{o}(F)\right)$ for $F \bar{F}= \pm 1$ ) is defined as the universal unital $C^{*}$-algebra generated by all the entries of a unitary $n$ by $n$ matrix $u=\left(u_{i, j}\right)_{i, j}$ satisfying

$$
\text { - } F \bar{u} F^{-1} \text { is unitary (respectively, } F \bar{u} F^{-1}=u \text { ), }
$$

where $\bar{u}=\left(u_{i, j}^{*}\right)_{i, j}$. Following 34] and [33, we treat only the case $n \geq 3$.

Put $\mathbb{G}:=A_{o}(F)$ or $A_{u}(F)$. Then $C(\mathbb{G})_{\text {red }}$ is defined as an image of $C(\mathbb{G})$ in $\mathbb{B}\left(L^{2}(\mathbb{G})\right)$ via the GNS-representation, and it is still a compact quantum group (with the Haar state faithful). Write $L^{\infty}(\mathbb{G}):=C(\mathbb{G})_{\text {red }}^{\prime \prime}$. Since the previous two representations $\lambda$ and $\rho$ are unitarily equivalent, we naturally have

$$
C(\mathbb{G})_{\mathrm{red}} \simeq \lambda(C(\mathbb{G})) \simeq \rho(C(\mathbb{G})), \quad L^{\infty}(\mathbb{G}) \simeq \lambda(C(\mathbb{G}))^{\prime \prime} \simeq \rho(C(\mathbb{G}))^{\prime \prime} .
$$

We regard $\rho(C(\mathbb{G})) \subset \rho(C(\mathbb{G}))^{\prime \prime} \subset \mathbb{B}\left(L^{2}(\mathbb{G})\right)$ as our main objects, and in the next section, we will prove that they satisfy some conditions similar to condition (AO). We note that factoriality and these types were studied in [2] and 34] (but not solved completely).

All the irreducible representations of $A_{o}(F)$ and $A_{u}(F)$ were completely classified in the following sense [3], 2]: $\operatorname{Irred}\left(A_{o}(F)\right)$ is identified with $\mathbb{N}$ in such a way that

$$
x \otimes y \simeq|x-y| \oplus(|x-y|+2) \oplus \cdots \oplus(x+y) \quad(x, y \in \mathbb{N}) ;
$$


$\operatorname{Irred}\left(A_{u}(F)\right)$ is identified with $\mathbb{N} * \mathbb{N}$ in such a way that

$$
x \otimes y \simeq \bigoplus_{z \in \mathbb{N} * \mathbb{N}, x=x_{0} z, y=\bar{z} y_{0}} x_{0} y_{0} \quad(x, y \in \mathbb{N} * \mathbb{N}) .
$$

From now on, for simplicity, we treat only $A_{o}(F)$, and all the cases of $A_{u}(F)$ in this paper can be treated in the same way as that of $A_{o}(F)$ (see [33, Section 5]).

Let $z$ be any irreducible representation contained in $x \otimes y$ as a subrepresentation (write as $z \in x \otimes y$ ). Let $p_{z}^{x \otimes y}$ be the unique projection in $\mathbb{B}\left(H_{x} \otimes H_{y}\right)$ satisfying $\left(U^{x} \otimes U^{y}\right)\left(p_{z}^{x \otimes y} \otimes 1\right) \simeq U^{z}$. Then take an intertwiner $V(x \otimes y, z)$ between $\left(U^{x} \otimes U^{y}\right)\left(p_{z}^{x \otimes y} \otimes 1\right)$ and $U^{z}$; it is unique up to multiplication by $\mathbb{T}$. Define a u.c.p. $\operatorname{map} \psi_{x+y, x}: \mathbb{B}\left(H_{x}\right) \rightarrow \mathbb{B}\left(H_{x+y}\right)$ by

$$
\psi_{x+y, x}(A):=V(x \otimes y, x+y)^{*}(A \otimes 1) V(x \otimes y, x+y),
$$

and note that this map does not depend on the choice of $V(x \otimes y, x+y)$.

Finally we recall a nuclear $C^{*}$-subalgebra $\mathcal{B}$ of $\ell^{\infty}(\hat{\mathbb{G}})$ which plays a significant role for us. We first put

$$
\mathcal{B}_{0}:=\left\{a \in \ell^{\infty}(\hat{\mathbb{G}}) \mid \text { there exists } x \text { such that } a p_{y}=\psi_{y, x}\left(a p_{x}\right) \text { for all } y \geq x\right\} .
$$

Let $\pi: \mathbb{B}\left(L^{2}(\mathbb{G})\right) \rightarrow \mathbb{B}\left(L^{2}(\mathbb{G})\right) / \mathbb{K}\left(L^{2}(\mathbb{G})\right)$ be the quotient map. In 34, Vaes and Vergnioux proved that

- the norm closure $\mathcal{B}$ of $\mathcal{B}_{0}$ is a $C^{*}$-algebra containing $c_{0}(\hat{\mathbb{G}})$ so that the $C^{*}$-algebra $\mathcal{B}_{\infty}:=\mathcal{B} / c_{0}(\hat{\mathbb{G}})$ is defined;

- $\mathcal{B}$ and $\mathcal{B}_{\infty}$ are nuclear;

- $\hat{\Phi}$ induces a left action of $\hat{\mathbb{G}}$ on $\mathcal{B}$ and $\mathcal{B}_{\infty}$;

- this left action on $\mathcal{B}_{\infty}$ is amenable so that $\mathcal{B}_{\infty} \rtimes_{r} \hat{\mathbb{G}}$ is nuclear and $\mathcal{B}_{\infty} \rtimes_{r} \hat{\mathbb{G}}=$ $\mathcal{B}_{\infty} \rtimes_{\text {full }} \hat{\mathbb{G}}$

- $\hat{\Phi}$ induces the trivial right action of $\hat{\mathbb{G}}$ on $\mathcal{B}_{\infty}$ so that $\hat{\lambda}\left(\mathcal{B}_{\infty}\right)$ commutes with $\pi \circ \lambda(C(\mathbb{G}))$, where we identify $\hat{\lambda}$ as a map from $\ell^{\infty}(\mathbb{G}) / c_{0}(\mathbb{G})$ to $\mathbb{B}\left(L^{2}(\mathbb{G})\right) / \mathbb{K}\left(L^{2}(\mathbb{G})\right)$.

Since $\hat{\lambda}: \mathcal{B}_{\infty} \rightarrow \mathbb{B}\left(L^{2}(\mathbb{G})\right) / \mathbb{K}\left(L^{2}(\mathbb{G})\right)$ and $(\iota \otimes \pi \circ \rho)(\mathbb{V}) \in M\left(c_{0}(\hat{\mathbb{G}}) \otimes \mathbb{B} / \mathbb{K}\right)$ form a covariant representation for the left action $\hat{\Phi}$, we have the $*$-homomorphism

$$
\pi_{l}(=\hat{\lambda} \rtimes \pi \circ \rho): \mathcal{B}_{\infty} \rtimes_{r} \hat{\mathbb{G}}=\mathcal{B}_{\infty} \rtimes_{\text {full }} \hat{\mathbb{G}} \longrightarrow \mathbb{B}\left(L^{2}(\mathbb{G})\right) / \mathbb{K}\left(L^{2}(\mathbb{G})\right)
$$

by universality. Putting $\pi_{r}:=\operatorname{Ad} U \circ \pi_{l}$, where $U=J \hat{J}$, we have the algebraic *-homomorphism

$$
\begin{aligned}
\pi_{l} \times \pi_{r}:\left(\mathcal{B}_{\infty} \rtimes_{r} \hat{\mathbb{G}}\right) \odot\left(\mathcal{B}_{\infty} \rtimes_{r} \hat{\mathbb{G}}\right) & \longrightarrow \mathbb{B}\left(L^{2}(\mathbb{G})\right) / \mathbb{K}\left(L^{2}(\mathbb{G})\right) \\
a \otimes b & \longmapsto \quad \pi_{l}(a) \pi_{r}(b),
\end{aligned}
$$

since $\hat{\lambda}\left(\mathcal{B}_{\infty}\right)$ commutes with $\pi \circ \lambda(C(\mathbb{G}))$. Here $\odot$ means the algebraic tensor product. By nuclearity of $\mathcal{B}_{\infty} \rtimes_{r} \hat{\mathbb{G}}$, this map is min-bounded, and the restriction of the map on $\left(\mathbb{C} \rtimes_{r} \hat{\mathbb{G}}\right) \otimes\left(\mathbb{C} \rtimes_{r} \hat{\mathbb{G}}\right) \simeq C(\mathbb{G})_{\text {red }} \otimes C(\mathbb{G})_{\text {red }}$ gives the min-boundedness of the multiplication map on $C(\mathbb{G})_{\text {red }}$ after taking the quotient with $\mathbb{K}\left(L^{2}(\mathbb{G})\right)$. This is the proof of the fact that $L^{\infty}(\mathbb{G})$ satisfies condition $(\mathrm{AO})$ given in 34 .

We note that the multiplication map from $C(\mathbb{G})_{\text {red }} \otimes C(\mathbb{G})_{\text {red }}$ to $\mathbb{B}\left(L^{2}(\mathbb{G})\right) /$ $\mathbb{K}\left(L^{2}(\mathbb{G})\right)$ is nuclear, since so is $\left(\mathcal{B}_{\infty} \rtimes_{r} \hat{\mathbb{G}}\right) \otimes\left(\mathcal{B}_{\infty} \rtimes_{r} \hat{\mathbb{G}}\right)$ (and hence so is $\left.\pi_{l} \times \pi_{r}\right)$. We will use this observation in the next section.

We finally mention that condition (AO) for $L^{\infty}\left(A_{o}(F)\right)$ and $L^{\infty}\left(A_{u}(F)\right)$ was first observed by Vergnioux in 32 . 


\section{Conditions similar to Ozawa's condition (AO)}

In this section, we introduce some similar conditions to condition (AO). We will prove that von Neumann algebras of $A_{o}(F)$ and $A_{u}(F)$ satisfy these conditions.

3.1. Condition $(\mathbf{A O})^{+}$. Let us first recall Ozawa's condition $(\mathrm{AO})$. We say a von Neumann algebra $M \subset \mathbb{B}(H)$ satisfies condition (AO) if there exist $\sigma$-weakly dense unital $C^{*}$-subalgebras $A \subset M$ and $B \subset M^{\prime}$ such that

(i) $A$ is locally reflexive;

(ii) the multiplication map $\nu: A \odot B \rightarrow \mathbb{B}(H) / \mathbb{K}(H) ; a \otimes b \mapsto a b+\mathbb{K}(H)$ is min-bounded.

In [23], Ozawa proved his celebrated theorem: if a finite von Neumann algebra satisfies condition (AO), then it is solid. As we mentioned, solidity (or semisolidity) implies primeness for non-injective $\mathrm{II}_{1}$ factors.

The most important examples of von Neumann algebras with condition (AO) come from bi-exact groups [4, Definition 15.1.2]. In fact, Ozawa proved that they have the following characterization [4, Lemma 15.1.4]: a countable discrete group $\Gamma$ is bi-exact if and only if $\Gamma$ is exact and satisfies the following condition:

- there exists a u.c.p. map $\theta: C_{\lambda}^{*}(\Gamma) \otimes C_{\rho}^{*}(\Gamma) \rightarrow \mathbb{B}\left(\ell^{2}(\Gamma)\right)$ such that $\theta(a \otimes b)-$ $a b \in \mathbb{K}\left(\ell^{2}(\Gamma)\right)$ for any $a \in C_{\lambda}^{*}(\Gamma)$ and $b \in C_{\rho}^{*}(\Gamma)$.

It is now obvious that the group von Neumann algebras of bi-exact groups satisfy condition (AO). Thus Ozawa proved that factors of bi-exact non-amenable i.c.c. groups are solid, in particular, prime.

Here is another significant viewpoint. To see solidity, we do not need the existence of a u.c.p. map $\theta$ above. We need only the property that the multiplication map $\nu$ is min-bounded after taking the quotient with $\mathbb{K}\left(\ell^{2}(\Gamma)\right)$. This is why condition $(\mathrm{AO})$ is weaker than bi-exactness for group von Neumann algebras.

On the other hand, in 27, Popa and Vaes proved that the group von Neumann algebras of bi-exact and weakly amenable groups are strongly solid. In the proof, they used such a u.c.p. map $\theta$ as an essential tool.

Motivated by these observations, we define the first condition similarly to condition (AO) as follows.

Definition 3.1.1. Let $M \subset \mathbb{B}(H)$ be a von Neumann algebra with standard representation and denote by $J$ the modular conjugation. We say $M \subset \mathbb{B}(H)$ satisfies condition $(\mathrm{AO})^{+}$if there exists a unital $\sigma$-weakly dense $C^{*}$-subalgebra $A$ such that

(i) $A$ is locally reflexive;

(ii) there exists a u.c.p. map $\theta: A \otimes J A J \rightarrow \mathbb{B}(H)$ such that $\theta(a \otimes J b J)-a J b J \in$ $\mathbb{K}(H)$ for any $a, b \in A$.

The difference of conditions $(\mathrm{AO})$ and $(\mathrm{AO})^{+}$is of course the existence of a u.c.p. map $\theta$. So it may be useful to consider how we get such a $\theta$ for von Neumann algebras satisfying condition (AO). For this purpose, we translate the second condition as follows.

(ii') The multiplication map $\nu$ is min-bounded and it has a u.c.p. lift; that is, there exists a u.c.p. map $\theta: A \otimes J A J \rightarrow \mathbb{B}(H)$ such that $\nu=\pi \circ \theta$, where $\pi: \mathbb{B}(H) \rightarrow \mathbb{B}(H) / \mathbb{K}(H)$ is the quotient map.

With this trivial translation, we can apply lifting theorems in some concrete cases. For example, if $A$ is a separable $C^{*}$-algebra and the multiplication map $\nu$ is nuclear, 
then $\nu$ has a u.c.p. lift by the lifting theorem due to Choi and Effros [6]. This method has been used by Ozawa (see the proof of [4, Proposition 15.2.3]).

Now combined with the observation in Subsection 2.4, we can easily deduce that our main targets satisfy condition $(\mathrm{AO})^{+}$.

Proposition 3.1.2. Von Neumann algebras $L^{\infty}\left(A_{o}(F)\right)$ and $L^{\infty}\left(A_{u}(F)\right)$ for $F \in$ $\mathrm{GL}(n, \mathbb{C})(n \geq 3)$ satisfy condition $(\mathrm{AO})^{+}$.

3.2. A similar condition for continuous cores. To see strong solidity in the rigidity theory, the finiteness assumption is essential since all the known proofs require the theory of amenable trace, which works only for finite von Neumann algebras. However our main targets $L^{\infty}\left(A_{o}(F)\right)$ and $L^{\infty}\left(A_{u}(F)\right)$ are hardly finite. So it is natural for us to see the continuous cores of such factors which are always semifinite.

In this subsection, we investigate some conditions for continuous cores of general von Neumann algebras. The following condition is a natural analogue of condition $(\mathrm{AO})^{+}$for continuous cores.

Definition 3.2.1. Let $M$ be a von Neumann algebra, $\phi$ a faithful normal state on $M$, and $\tilde{J}$ the modular conjugation for $M \rtimes_{\sigma^{\phi}} \mathbb{R} \subset \mathbb{B}\left(L^{2}(M, \phi) \otimes L^{2}(\mathbb{R})\right)$. We say the pair $(M, \phi)$ satisfies condition $(\mathrm{AO})^{+}$with respect to its continuous core (say condition $(\mathrm{AOC})^{+}$, for short) if there exists a $\sigma$-weakly dense unital $C^{*}$-subalgebra $A \subset M$ such that

(i) $\sigma^{\phi}$ defines a norm continuous action on $A$ (so that we can define $A \rtimes_{r} \mathbb{R}$ );

(ii) $A \rtimes_{r} \mathbb{R}$ is locally reflexive;

(iii) there exists a u.c.p. map

$$
\begin{gathered}
\theta:\left(A \rtimes_{r} \mathbb{R}\right) \odot \tilde{J}\left(A \rtimes_{r} \mathbb{R}\right) \tilde{J} \longrightarrow \mathbb{B}\left(L^{2}(M, \phi) \otimes L^{2}(\mathbb{R})\right) \\
\text { such that } \theta(a \otimes \tilde{J} b \tilde{J})-a \tilde{J} b \tilde{J} \in \mathbb{K}\left(L^{2}(M, \phi)\right) \otimes \mathbb{B}\left(L^{2}(\mathbb{R})\right) \text { for any } a, b \in A \rtimes_{r} \mathbb{R} .
\end{gathered}
$$

Our goal in this subsection is to show that $A_{o}(F)$ and $A_{u}(F)$ with the Haar states satisfy this condition. For this, we investigate a sufficient condition for condition $(\mathrm{AOC})^{+}$.

Let $M$ be a von Neumann algebra and $\phi$ a faithful normal state on $M$. Write $H:=L^{2}(M, \phi)$ and $\mathcal{K}:=\mathbb{K}(H) \otimes \mathbb{B}\left(L^{2}(\mathbb{R})\right)$ and let $J$ be the modular conjugation on $H$. Consider the multiplier algebra $\mathcal{L}:=M(\mathcal{K})$ of $\mathcal{K}$ and denote $\mathcal{C}:=\mathcal{L} / K$.

Assume first that there exists a $\sigma$-weakly dense unital $C^{*}$-subalgebra $A \subset M$ such that

(a) $\sigma^{\phi}$ defines a norm continuous action on $A$ (so that we can define $A \rtimes_{r} \mathbb{R}$ ).

Let $\pi$ be a $*$-homomorphism from $\mathbb{B}(H)$ into $\mathbb{B}\left(H \otimes L^{2}(\mathbb{R})\right)$ given by $(\pi(x) \xi)(t):=$ $\Delta_{\phi}^{-i t} x \Delta_{\phi}^{i t} \xi(t)$ for $x \in \mathbb{B}(H)$ and $t \in \mathbb{R}$. Consider the $C^{*}$-algebra $D$ generated by the following elements:

- $\pi(a), J b J \otimes 1(a, b \in A)$;

- $1 \otimes \lambda_{t}, \Delta_{\phi}^{i t} \otimes \rho_{t}(t \in \mathbb{R})$;

- $\int_{\mathbb{R}} f(s)\left(1 \otimes \lambda_{s}\right) \cdot d s, \int_{\mathbb{R}} f(s)\left(\Delta_{\phi}^{i s} \otimes \rho_{s}\right) \cdot d s\left(f \in L^{1}(\mathbb{R})\right)$.

Then we assume that

(b) $D$ is contained in $\mathcal{L}$.

In particular, we have natural maps from $A$ and $A^{\mathrm{op}}(=J A J)$ to $\mathcal{C}$. We denote these maps by $\pi_{l}$ and $\pi_{r}$ respectively. 
Next we assume that

(c) there exist separable nuclear $C^{*}$-algebras $C_{l}$ and $C_{r}$ containing $A$ and $A^{\text {op }}$ respectively (so that $A$ is exact);

(d) there exist *-homomorphisms from $C_{l}$ and $C_{r}$ to $\mathcal{C}$ such that they are extensions of $\pi_{l}$ and $\pi_{r}$, respectively. We still denote them by $\pi_{l}$ and $\pi_{r}$.

Then we want to define the following $*$-homomorphism:

$$
\nu: C_{l} \odot C_{r} \longrightarrow \mathcal{C} ; a \otimes b \mapsto \pi_{l}(a) \pi_{r}(b) .
$$

However we do not know whether ranges of $C_{l}$ and $C_{r}$ commute, and hence we further assume that

(e) $\nu$ is a well-defined $*$-homomorphism, that is, $\left[\pi_{l}(a), \pi_{r}(b)\right]=0\left(a \in C_{l}\right.$, $\left.b \in C_{r}\right)$.

We can extend $\nu$ on $C_{l} \otimes C_{r}$ by the nuclearity. Restricting this map, we have a natural multiplication $*$-homomorphism

$$
\begin{aligned}
& \nu: A \otimes A^{\mathrm{op}} \longrightarrow \mathcal{C} ; a \otimes 1 \\
& 1 \otimes a^{\mathrm{op}} \longmapsto\left[a^{\mathrm{op}} \otimes 1\right] .
\end{aligned}
$$

Next consider norm continuous $(\mathbb{R} \times \mathbb{R})$-actions on $A \otimes A^{\text {op }}$ and $\nu\left(A \otimes A^{\text {op }}\right)$ given by

$$
\begin{aligned}
& \mathbb{R} \times \mathbb{R} \longrightarrow \operatorname{Aut}\left(A \otimes A^{\mathrm{op}}\right) \quad ; s \otimes t \longmapsto \quad \sigma_{s}^{\phi} \otimes \tilde{\sigma}_{t}^{\phi}, \\
& \mathbb{R} \times \mathbb{R} \longrightarrow \operatorname{Aut}\left(\nu\left(A \otimes A^{\mathrm{op}}\right)\right) ; s \otimes t \longmapsto \operatorname{Ad}\left(\left[1 \otimes \lambda_{s}\right]\left[\Delta_{\phi}^{i t} \otimes \rho_{t}\right]\right) .
\end{aligned}
$$

Here $\tilde{\sigma}_{t}^{\phi}\left(a^{\mathrm{op}}\right)=\tilde{\sigma}_{t}^{\phi}\left(J a^{*} J\right):=J \sigma_{t}^{\phi}\left(a^{*}\right) J=\sigma_{t}^{\phi}(a)^{\mathrm{op}}$. It is easily verified that $\nu$ is $(\mathbb{R} \times \mathbb{R})$-equivariant and hence we have the following $*$-homomorphism:

$\tilde{\nu}: A \rtimes_{r} \mathbb{R} \otimes \tilde{J}\left(A \rtimes_{r} \mathbb{R}\right) \tilde{J} \simeq\left(A \otimes A^{\mathrm{op}}\right) \rtimes_{r}(\mathbb{R} \times \mathbb{R}) \rightarrow\left(\nu\left(A \otimes A^{\mathrm{op}}\right)\right) \rtimes_{r}(\mathbb{R} \times \mathbb{R}) \rightarrow \mathcal{C}$.

Here the continuity of the final map comes from the amenability of $\mathbb{R} \times \mathbb{R}$. The resulting map says that the multiplication map on $A \rtimes_{r} \mathbb{R} \odot\left(A \rtimes_{r} \mathbb{R}\right)^{\text {op }}$ to $\mathcal{L} \subset$ $\mathbb{B}\left(H \otimes L^{2}(\mathbb{R})\right)$ is min-bounded after taking the quotient with $\mathcal{K}$. Now the $C^{*}$-algebra $A \rtimes_{r} \mathbb{R}$ is exact (and hence locally reflexive) since so is $A$, and it is $\sigma$-weakly dense in $M \rtimes_{\sigma^{\phi}} \mathbb{R}$. At this point, $M \rtimes_{\sigma^{\phi}} \mathbb{R}$ satisfies a similar condition to condition (AO).

Finally we assume that

(f) $\operatorname{Ad}\left(\left[1 \otimes \lambda_{s}\right]\left[\Delta_{\phi}^{i t} \otimes \rho_{t}\right]\right) \nu\left(C_{l} \otimes C_{r}\right)=\nu\left(C_{l} \otimes C_{r}\right)$ for any $(s, t) \in \mathbb{R} \times \mathbb{R}$ and this defines a norm continuous action on $\nu\left(C_{l} \otimes C_{r}\right)$.

In this case there exists a $*$-homomorphism from $\nu\left(C_{l} \otimes C_{r}\right) \rtimes_{r}(\mathbb{R} \times \mathbb{R})$ into $\mathcal{C}$ and hence the image of the map is nuclear. Since ran $\tilde{\nu}$ is contained in this image, $\tilde{\nu}$ is a nuclear map into $\mathcal{C}$. Thus the lifting theorem of Choi and Effros is again applicable so that $\tilde{\nu}$ has a u.c.p. lift. In summary we have the following lemma.

Lemma 3.2.2. Let $M$ be a von Neumann algebra, $\phi$ a faithful normal state on $M$, and $A \subset M$ a $\sigma$-weakly dense unital $C^{*}$-subalgebra. If they satisfy all the conditions from (a) to (f), then $(M, \phi)$ satisfies condition $(\mathrm{AOC})^{+}$.

Now we turn to showing that our main objects satisfy these conditions.

Proposition 3.2.3. Von Neumann algebras $L^{\infty}\left(A_{o}(F)\right)$ and $L^{\infty}\left(A_{u}(F)\right)$ for $F \in$ $\mathrm{GL}(n, \mathbb{C})(n \geq 3)$ with the Haar state $h$ satisfy condition $(\mathrm{AOC})^{+}$. 
Proof. We keep the notation in Subsection 2.4, Put $A:=C_{\text {red }}^{*}(\mathbb{G})=\rho(C(\mathbb{G})) \subset$ $\mathbb{B}\left(L^{2}(\mathbb{G})\right)$ and $C_{l}=C_{r}=\mathcal{B}_{\infty} \rtimes_{r} \mathbb{G}$. We will verify all the conditions from (a) to (f) above. Note that the condition (a) is a well-known property.

For this, recall the following formula: for any irreducible decomposition $x \otimes y \simeq$ $\sum_{z \in x \otimes y} \oplus z$, we have

$$
F_{x} \otimes F_{y} \simeq \sum_{z \in x \otimes y} \bigoplus F_{z} \quad \text { on } H_{x} \otimes H_{y} \simeq \sum_{z \in x \otimes y} \bigoplus H_{z}
$$

Indeed this follows from a direct calculation of $\left(\iota \otimes \iota \otimes f_{1}\right)\left(U_{13}^{x} U_{23}^{y}\right)$. By the formula, we have the following relation:

$$
\Delta^{i t} \hat{\lambda}\left(\psi_{x+y, x}(B)\right) \Delta^{-i t}=\hat{\lambda}\left(\psi_{x+y, x}\left(F_{x}^{i t} B F_{x}^{-i t}\right)\right) \quad\left(B \in \mathbb{B}\left(H_{x}\right)\right) .
$$

In this sense, the modular group $\Delta^{i t}$ commutes with all $\psi_{x+y, y}$.

To see the condition (b), observe first that for any $c \in \mathbb{B}\left(H \otimes L^{2}(\mathbb{R})\right)$, $c$ is contained in $\mathcal{K}$ if and only if

$$
\left\|\sum_{x=0}^{y}\left(p_{x} \otimes 1\right) c \sum_{x=0}^{z}\left(p_{x} \otimes 1\right)-c\right\| \rightarrow 0 \quad(y, z \rightarrow \infty) .
$$

Let $a$ be an element in $\mathcal{K}$ and $b$ a generator of $D$. We will show $b a \in \mathcal{K}$. The cases $b=1 \otimes \lambda_{t}, \Delta_{\phi}^{i t} \otimes \rho_{t}$, and $d \otimes 1\left(d \in U A U^{*}\right)$ are trivial. The cases $b=\int_{\mathbb{R}} f(s)\left(1 \otimes \lambda_{s}\right) \cdot d s$ and $\int_{\mathbb{R}} f(s)\left(\Delta_{\phi}^{i s} \otimes \rho_{s}\right) \cdot d s\left(f \in L^{1}(\mathbb{R})\right)$ are easy since they commute with all $p_{x}$. For the final case $b=\pi(d)(d \in A)$, we may assume $d=\left(\omega_{\xi, \eta} \otimes \rho\right)(\mathbb{V})$ for $\xi, \eta \in H_{z}$ and $z \in \operatorname{Irred}(\mathbb{G})$. Let $\left(\xi_{k}^{z}\right)_{k=1}^{n_{z}}$ be a fixed orthonormal basis of $H_{z}$. Then we have

$$
\begin{aligned}
p_{x} \sigma_{t}(d) & =p_{x} \sigma_{t}\left(\left(\omega_{\xi, \eta} \otimes \rho\right)(\mathbb{V})\right) \\
& =\left(\omega_{\xi, \eta} \otimes \iota\right)\left(\left(1 \otimes p_{x}\right) \mathscr{V}_{t}\right) \quad\left(\mathscr{V}_{t}:=\left(\iota \otimes \sigma_{t} \circ \rho\right)(\mathbb{V})\right) \\
& =\left(\omega_{\xi, \eta} \otimes \iota\right)\left(\mathscr{V}_{t} \mathscr{V}_{t}^{*}\left(1 \otimes p_{x}\right) \mathscr{V}_{t}\right) \\
& =\sum_{k=1}^{n_{z}}\left(\omega_{\xi, \xi_{k}^{z}} \otimes \iota\right)\left(\mathscr{V}_{t}\right)\left(\omega_{\xi_{k}^{z}, \eta} \otimes \iota\right)\left(\left(1 \otimes \Delta^{i t}\right) \hat{\Psi}\left(p_{x}\right)\left(1 \otimes \Delta^{-i t}\right)\right)
\end{aligned}
$$

for all $x \in \operatorname{Irred}(\mathbb{G})$, where $\sigma_{t}$ is the modular group for the Haar state and $\hat{\Psi}\left(p_{x}\right):=$ $(\iota \otimes \rho)(\mathbb{V})^{*}\left(1 \otimes p_{x}\right)(\iota \otimes \rho)(\mathbb{V})$. Since $\sum_{x=0}^{y} p_{x}$ converges to 1 in the strong topology as $y \rightarrow \infty$ and $\hat{\Psi}$ is normal, each $\left(\omega_{\xi_{k}^{z}, \eta} \otimes \iota\right)\left(\left(1 \otimes \Delta^{i t}\right) \hat{\Psi}\left(\sum_{x=0}^{y} p_{x}\right)\left(1 \otimes \Delta^{-i t}\right)\right)$ converges to $\omega_{\xi_{k}^{z}, \eta}(1) 1$ in the strong topology. Hence for any compact operator $T \in \mathbb{K}(H)$, the equation

$$
\sum_{x=0}^{y} p_{x} \sigma_{t}(d) T=\sum_{k=1}^{n_{k}}\left(\omega_{\xi, \xi_{k}^{z}} \otimes \iota\right)\left(\mathscr{V}_{t}\right)\left(\omega_{\xi_{k}^{z}, \eta} \otimes \iota\right)\left(\left(1 \otimes \Delta^{i t}\right) \hat{\Psi}\left(\sum_{x=0}^{y} p_{x}\right)\left(1 \otimes \Delta^{-i t}\right)\right) T
$$

implies that $\sum_{x=0}^{y} p_{x} \sigma_{t}(d) T$ converges to $\sigma_{t}(d) T$ in the norm topology as $y \rightarrow \infty$. We choose $T$ as an element of the set of all linear combinations of the form $\xi_{k}^{a} \otimes \xi_{l}^{b}$ for $a, b \in \operatorname{Irred} \mathbb{G}$ and $k, l$. Then it is easy to verify that this convergence is uniform with respect to $t \in \mathbb{R}$, that is,

$$
\sup _{t \in \mathbb{R}}\left\|\left(1-\sum_{x=0}^{y} p_{x}\right) \sigma_{t}(d) T\right\| \rightarrow 0 \quad(y \rightarrow \infty) .
$$


Now, for any $S \in \mathbb{B}\left(L^{2}(\mathbb{R})\right)$, we have

$$
\begin{aligned}
& \left\|\sum_{x=0}^{y}\left(p_{x} \otimes 1\right) \pi(d)(T \otimes S)-\pi(d)(T \otimes S)\right\| \\
= & \left\|\int_{\mathbb{R}}\left(\sum_{x=0}^{y} p_{x}-1\right) \sigma_{-t}(d) \otimes e_{t} \cdot d t(T \otimes S)\right\| \\
\leq & \sup _{t \in \mathbb{R}}\left\|\left(\sum_{x=0}^{y} p_{x}-1\right) \sigma_{t}(d) T\right\|\|S\| \rightarrow 0 \quad(y \rightarrow \infty) .
\end{aligned}
$$

Hence, for any $a \in \mathcal{K}=\mathbb{K}(H) \otimes \mathbb{B}\left(L^{2}(\mathbb{R})\right)$, we have

$$
\left\|\sum_{x=0}^{y}\left(p_{x} \otimes 1\right) \pi(d) a-\pi(d) a\right\| \rightarrow 0 \quad(y \rightarrow \infty) .
$$

Since $\pi(d) a \sum_{x=0}^{y}\left(p_{x} \otimes 1\right)$ converges to $\pi(d) a$ in the norm topology, $\pi(d) a$ is contained in $\mathcal{K}$, and we get the condition (b).

Next we define two maps $\pi_{l}$ and $\pi_{r}$. We begin with the following maps:

$$
\begin{aligned}
& \mathcal{B} \longrightarrow \mathcal{L} ; a \longmapsto \pi(\hat{\lambda}(a)), \\
& \mathcal{B} \longrightarrow \mathcal{L} ; a \longmapsto U \hat{\lambda}(a) U^{*} \otimes 1 .
\end{aligned}
$$

It is not difficult to see that ranges of these maps are really contained in $\mathcal{L}$. Since images of $c_{0}(\hat{\mathbb{G}})$ by these maps are contained in $\mathcal{K}$, we have induced maps from $\mathcal{B}_{\infty}$ to $\mathcal{C}$. Simple calculations show that these maps make two covariant representations of $\mathcal{B}_{\infty}$ and the natural left action of $\hat{\mathbb{G}}$. Since this action is amenable we have the following desired maps:

$$
\begin{aligned}
\pi_{l}: C_{l} & =\mathcal{B}_{\infty} \rtimes_{r} \hat{\mathbb{G}} \longrightarrow \mathcal{C}, \\
\pi_{r}: C_{r} & =\mathcal{B}_{\infty} \rtimes_{r} \hat{\mathbb{G}} \longrightarrow \mathcal{C} .
\end{aligned}
$$

Finally we prove the condition (e) (and then the condition (f) is easily verified). For this, it suffices to see that $\pi \circ \hat{\lambda}(\mathcal{B})$ (respectively, $\hat{\rho}(\mathcal{B}) \otimes 1$ ) commutes with $\lambda(C(\mathbb{G})) \otimes 1$ (respectively, $\pi \circ \rho(C(\mathbb{G}))$ ) after taking the quotient with $\mathcal{K}$. Here we treat only the case of $\pi \circ \hat{\lambda}(\mathcal{B})$ and $\lambda(C(\mathbb{G})) \otimes 1$, and the other case follows in the same manner.

Let $z$ be an element of $\operatorname{Irred}(\mathbb{G})$ and write as $U^{z}=\sum_{i, j} u_{i, j}^{z} \otimes e_{i, j}$, where $\left(e_{i j}\right)_{i j}$ is a fixed matrix unit in $\mathbb{B}\left(H_{z}\right)$. Our goal is to show that

$$
\left[\pi \circ \hat{\lambda}(b), \lambda\left(u_{i, j}^{z}\right) \otimes 1\right] \in \mathbb{K}\left(L^{2}(\mathbb{G})\right) \otimes \mathbb{B}\left(L^{2}(\mathbb{R})\right)
$$

for any $z, i, j$ and any $b \in \mathcal{B}$, where $[\cdot, \cdot]$ is the commutator. Since this term coincides with

$$
\int_{\mathbb{R}}\left[\Delta^{-i t} \hat{\lambda}(b) \Delta^{i t}, \lambda\left(u_{i, j}^{z}{ }^{*}\right)\right] \otimes e_{t} \cdot d t,
$$

running over all $i$ and $j$, our goal is equivalent to

$$
\int_{\mathbb{R}} \sum_{i, j}\left[\Delta^{-i t} \hat{\lambda}(b) \Delta^{i t}, \lambda\left(u_{i, j}^{z}{ }^{*}\right)\right] \otimes e_{i, j} \otimes e_{t} \cdot d t \in \mathbb{K}\left(L^{2}(\mathbb{G})\right) \otimes \mathbb{B}\left(H_{z}\right) \otimes \mathbb{B}\left(L^{2}(\mathbb{R})\right),
$$


and using $(\lambda \otimes \hat{\lambda})\left(\mathbb{V}_{21}^{*}\right)\left(1 \otimes p_{z}\right)=\sum_{i, j} u_{i, j}^{z}{ }^{*} \otimes e_{i, j} \otimes \operatorname{id}_{H_{\bar{z}}}\left(\right.$ write $\left.W:=(\lambda \otimes \hat{\lambda})\left(\mathbb{V}_{21}\right)\right)$, we further translate it as

$$
\int_{\mathbb{R}}\left[\Delta^{-i t} \hat{\lambda}(b) \Delta^{i t} \otimes p_{z}, W^{*}\left(1 \otimes p_{z}\right)\right] \otimes e_{t} \cdot d t \in \mathbb{K}\left(L^{2}(\mathbb{G})\right) \otimes \mathbb{B}\left(H_{z} \otimes H_{\bar{z}}\right) \otimes \mathbb{B}\left(L^{2}(\mathbb{R})\right) .
$$

For simplicity, we denote it by $\int_{\mathbb{R}} \mathrm{T}_{t} \otimes e_{t} \cdot d t$. If $b \in \mathcal{B}$ is finitely supported, that is, contained in a finite direct sum of $\mathbb{B}\left(H_{x}\right)(x \in \operatorname{Irred}(\mathbb{G}))$, then this final condition holds since this term is contained in $\left(\bigoplus_{\text {fin }} \mathbb{B}\left(H_{x} \otimes H_{\bar{x}}\right)\right) \otimes \mathbb{B}\left(H_{z} \otimes H_{\bar{z}}\right) \otimes \mathbb{B}\left(L^{2}(\mathbb{R})\right)$. Hence we may assume that $b=\psi_{\infty, x}(A) \in \mathcal{B}$ for some $A \in \mathbb{B}\left(H_{x}\right)$, where $\psi_{\infty, x}(A)$ is defined by $\psi_{\infty, x}(A) p_{y}:=\psi_{y, x}(A)$ if $y \geq x$ and 0 otherwise. have

Now by the proof of $[34$, Proposition 3.8], for any $y \in \operatorname{Irred}(\mathbb{G})$ with $y \geq z$ we

$$
\begin{aligned}
& \left\|\mathrm{T}_{t}\left(p_{x+y} \otimes p_{z}\right)\right\| \\
= & \left\|\left[\Delta^{-i t} \hat{\lambda}\left(\psi_{\infty, x}(A)\right) \Delta^{i t} \otimes 1, W^{*}\right]\left(p_{x+y} \otimes p_{z}\right)\right\| \\
= & \left\|\left[\hat{\lambda}\left(\psi_{\infty, x}\left(F_{x}^{-i t} A F_{x}^{i t}\right)\right) \otimes 1, W^{*}\right]\left(p_{x+y} \otimes p_{z}\right)\right\| \\
= & \left\|\left\{W\left(\hat{\lambda}\left(\psi_{\infty, x}\left(F_{x}^{-i t} A F_{x}^{i t}\right)\right) \otimes 1\right) W^{*}-\hat{\lambda}\left(\psi_{\infty, x}\left(F_{x}^{-i t} A F_{x}^{i t}\right)\right) \otimes 1\right\}\left(p_{x+y} \otimes p_{z}\right)\right\| \\
= & \left\|(\hat{\lambda} \otimes \hat{\lambda})\left\{\hat{\Phi}\left(\psi_{\infty, x}\left(F_{x}^{-i t} A F_{x}^{i t}\right) \otimes 1\right)-\psi_{\infty, x}\left(F_{x}^{-i t} A F_{x}^{i t}\right) \otimes 1\right\}\left(p_{x+y} \otimes p_{z}\right)\right\| \\
\leq & C(z)\left\|F_{x}^{-i t} A F_{x}^{i t}\right\| q^{y}=C(z)\|A\| q^{y},
\end{aligned}
$$

where $C(z)$ and $0<q<1$ are constants $(C(z)$ depends on $z)$. Since this estimate does not depend on $t \in \mathbb{R}$, we have the following norm convergent sequence:

$$
\int_{\mathbb{R}} \sum_{k=0}^{y}\left(\mathrm{~T}_{t}\left(p_{x+k} \otimes p_{z}\right)\right) \otimes e_{t} \cdot d t \rightarrow \int_{\mathbb{R}} \sum_{k=0}^{\infty}\left(\mathrm{T}_{t}\left(p_{x+k} \otimes p_{z}\right)\right) \otimes e_{t} \cdot d t \quad(y \rightarrow \infty) .
$$

Now each element in this sequence is contained in $\mathbb{K}\left(L^{2}(\mathbb{G})\right) \otimes \mathbb{B}\left(H_{z} \otimes H_{\bar{z}}\right) \otimes \mathbb{B}\left(L^{2}(\mathbb{R})\right)$ and the limit element coincides with $\int_{\mathbb{R}} \mathrm{T}_{t} \otimes e_{t} \cdot d t$. Hence we can end the proof.

\section{Absence of Cartan subalgebras}

In this section, we prove Theorems $\mathrm{A}$ and $\mathrm{B}$ in almost the same way as 27 , Theorem 3.1]. Since many proofs are the same, we often omit them.

4.1. Preparation with the $\mathbf{W}^{*} \mathbf{C B A P}$ and condition $(\mathbf{A O})^{+}$. Since we have similar arguments for proofs of both theorems, we first assume that $M$ is an arbitrary semifinite von Neumann algebra with separable predual; we will give other assumptions in each lemma.

Let $M$ be a semifinite von Neumann algebra with separable predual, Tr a faithful normal semifinite trace, $p$ a Tr-finite projection in $M$, and $A \subset p M p$ a von Neumann subalgebra. Write $P:=\mathcal{N}_{p M p}(A)^{\prime \prime}$. For simplicity we assume $\operatorname{Tr}(p)=1$. As usual, we identify $J_{M} M J_{M}$ and $J_{P} P J_{P}$ as opposite algebras $M^{\mathrm{op}}$ and $P^{\mathrm{op}}$ via natural identifications and write $\bar{a}:=\left(a^{\mathrm{op}}\right)^{*}\left(=J_{M} a J_{M}\right.$ or $\left.J_{P} a J_{P}\right)$ for $a \in M$ or $P$. Put $D:=M \odot M^{\mathrm{op}} \odot P^{\mathrm{op}} \odot P$ and define two $*$-homomorphisms:

$$
\begin{aligned}
& \Psi: D \longrightarrow \mathbb{B}\left(L^{2}(M) \otimes L^{2}(M) \otimes L^{2}(P)\right) ; a \otimes b^{\mathrm{op}} \otimes x^{\mathrm{op}} \otimes y \longmapsto a \otimes b^{\mathrm{op}} \otimes x^{\mathrm{op}} y, \\
& \Theta: D \longrightarrow \mathbb{B}\left(L^{2}(M) \otimes L^{2}(P)\right) \quad ; a \otimes b^{\mathrm{op}} \otimes x^{\mathrm{op}} \otimes y \longmapsto a b^{\mathrm{op}} \otimes x^{\mathrm{op}} y .
\end{aligned}
$$

The following theorem is due to Popa and Ozawa. In the theorem, $L^{2}(A), L^{2}(P)$, and $L^{2}(p M p)$ means GNS representations of $\operatorname{Tr}(p \cdot p)$. 
Theorem 4.1.1 ([24, [22]). If $p M p$ has the $W^{*} C B A P$ and $A$ is injective, then the conjugate action of $\mathcal{N}_{p M p}(A)$ on $A$ is weakly compact; that is, there exists a net $\left(\xi_{i}\right)_{i}$ of unit vectors in the positive cone of $L^{2}(A) \otimes L^{2}(A) \subset L^{2}(p M p) \otimes L^{2}(P)$ satisfying the following conditions:

(i) $\left\langle(x \otimes 1) \xi_{i}, \xi_{i}\right\rangle \rightarrow \operatorname{Tr}(x)$ for any $x \in p M p$;

(ii) $\left\|(a \otimes \bar{a}) \xi_{i}-\xi_{i}\right\| \rightarrow 0$ for any $a \in \mathcal{U}(A)$;

(iii) $\left\|\xi_{i}-((u \otimes \bar{u}) J(u \otimes \bar{u}) J) \xi_{i}\right\| \rightarrow 0$ for any $u \in \mathcal{N}_{p M p}(A)$, where $J:=J_{p M p} \otimes J_{P}$.

Note that regarding $\left(\xi_{i}\right)_{i}$ as vectors in $L^{2}(M, \operatorname{Tr}) \otimes L^{2}(P)$ by $L^{2}(p M p) \simeq$ $p J_{M} p J_{M} L^{2}(M, \operatorname{Tr}) \subset L^{2}(M, \operatorname{Tr})$, we get

$\left(\mathrm{i}^{\prime}\right)\left\langle(x \otimes 1) \xi_{i}, \xi_{i}\right\rangle \rightarrow \operatorname{Tr}(p x p)$ for any $x \in M$.

In our proof, we will use $\left(\xi_{i}\right)_{i}$ as vectors in the positive cone of $L^{2}(M) \otimes L^{2}(P)$ satisfying $\left(p J_{M} p J_{M} \otimes 1_{P}\right) \xi_{i}=\xi_{i}$ and conditions (i)', (ii), and (iii). In this case, we can exchange $L^{2}(M)$ and $L^{2}(P)$ with any other standard representations of $M$ and $P$, since these conditions are preserved under adjoint maps by canonical unitaries between standard representations.

We fix such a net $\left(\xi_{i}\right)_{i}$ and put $\Omega_{1}(x):=\operatorname{Lim}_{i}\left\langle x \xi_{i}, \xi_{i}\right\rangle$ for $x \in \mathbb{B}\left(L^{2}(M) \otimes L^{2}(P)\right)$, where Lim is taken by a fixed free ultrafilter. Then conditions (i'), (ii) and (iii) are translated as the following conditions:

(iv) $\Omega_{1}(x \otimes 1)=\operatorname{Tr}(p x p)$ for any $x \in M$;

(v) $\Omega_{1}(a \otimes \bar{a})=1$ for any $a \in \mathcal{U}(A)$;

(vi) $\Omega_{1}(\Theta(u \otimes \bar{u} \otimes \bar{u} \otimes u))=1$ for any $u \in \mathcal{N}_{p M p}(A)$.

We next prove the following lemma. In the original paper of Popa and Vaes, the proof of the corresponding statement is very technical (whose origin is in 9, Lemma $6.2]$ ). In the present paper, we give a very simple proof which works only in our special situation. We are indebted to Eric Ricard for kindly demonstrating this simple proof.

Lemma 4.1.2. Let $\Omega$ be a state on $\mathbb{B}\left(L^{2}(M) \otimes L^{2}(P)\right)$ satisfying condition (v) above. Assume that $A$ is diffuse. Then $\Omega(x \otimes 1)=0$ for any $x \in \mathbb{K}\left(L^{2}(M)\right)$. In particular, there exists an increasing net $\left(p_{j}\right)_{j}$ of range finite projections in $\mathbb{B}\left(L^{2}(M)\right)$ such that

- $p_{j} \rightarrow 1_{M}$ strongly;

- $\Omega\left(p_{j} \otimes 1_{P}\right)=0$ for any $p_{j}$.

Proof. Since $A$ is diffuse, we can find a sequence $\left(u_{n}\right)$ of unitaries in $A$ satisfying the following conditions:

- $\left(u_{n}\right)_{n}$ converges to 0 in the $\sigma$-weak topology;

- $\left(\frac{1}{n} \sum_{k=1}^{n} u_{k} p u_{k}^{*}\right)_{n}$ converges to 0 in the norm topology for any $p \in \mathbb{K}\left(L^{2}(B)\right)$.

By condition $(\mathrm{v}), \mathcal{U}(A)$ is contained in the multiplicative domain of $\Omega$ (e.g. 4, Proposition 1.5.7]). Since each $u_{n}$ is a unitary in $A$, we have, for any $p \in \mathbb{K}\left(L^{2}(M)\right)$,

$$
\begin{aligned}
\Omega\left(p \otimes 1_{P}\right) & =\Omega\left(u_{k} p u_{k}^{*} \otimes 1_{P}\right) \\
& =\Omega\left(\frac{1}{n} \sum_{k=1}^{n} u_{k} p u_{k}^{*} \otimes 1_{P}\right) \rightarrow 0 .
\end{aligned}
$$


Next, we assume that $M$ satisfies condition $(\mathrm{AO})^{+}$with a $\sigma$-weakly dense $C^{*}$ subalgebra $M_{0}$ and a u.c.p. map $\theta$. Put $D_{0}:=M_{0} \odot M_{0}^{\text {op }} \odot P^{\text {op }} \odot P \subset D$. We use the map $\theta$ only in the following simple lemma.

Lemma 4.1.3. Let $p_{j}$ be range finite projections in $\mathbb{B}\left(L^{2}(M)\right)$ with $p_{j} \rightarrow 1$ strongly and assume $M$ satisfies condition $(\mathrm{AO})^{+}$. Then we have $\lim \sup _{j}\left\|\Theta(S)\left(p_{j}^{\perp} \otimes 1\right)\right\| \leq$ $\|\Psi(S)\|$ for any $S \in D_{0}$, where $p_{j}^{\perp}:=1-p_{j}$.

Proof. For $S:=a \otimes b^{\text {op }} \otimes x^{\text {op }} \otimes y \in D_{0}$, we have

$$
(\theta \otimes \mathrm{id}) \circ \Psi(S)-\Theta(S)=\left(\theta\left(a \otimes b^{\mathrm{op}}\right)-a b^{\mathrm{op}}\right) \otimes x^{\mathrm{op}} y \in \mathbb{K}\left(L^{2}(M)\right) \otimes \mathbb{B}\left(L^{2}(P)\right) .
$$

Since $p_{j}^{\perp}$ converges to 0 in the strong topology and $\theta\left(a \otimes b^{\text {op }}\right)-a b^{\text {op }} \in \mathbb{K}\left(L^{2}(M)\right)$, the net $\left(\theta\left(a \otimes b^{\mathrm{op}}\right)-a b^{\mathrm{op}}\right) p_{j}^{\perp}$ converges to 0 in the norm topology. Hence we have

$$
\begin{aligned}
\limsup _{j}\left\|\Theta(S)\left(p_{j}^{\perp} \otimes 1\right)\right\| & \leq \limsup _{j}\left\|((\theta \otimes \mathrm{id}) \circ \Psi(S)-\Theta(S))\left(p_{j}^{\perp} \otimes 1\right)\right\|+\|\Psi(S)\| \\
& =\|\Psi(S)\| .
\end{aligned}
$$

This holds for any $S \in D_{0}$ in the completely same manner.

4.2. Proof of Theorem A. We prove the following theorem, which is slightly more general than Theorem $\mathrm{A}$

Theorem 4.2.1. Let $M$ be a semifinite von Neumann algebra with separable predual and $p$ a finite projection in $M$. If $M$ satisfies condition $(\mathrm{AO})^{+}$and has the $W^{*} C B A P$, then $p M p$ is strongly solid.

Proof. Let $A \subset p M p$ be a diffuse injective von Neumann subalgebra. Take $\left(\xi_{i}\right)_{i}$, $\Omega_{1} D, D_{0}, \Psi$, and $\Theta$ as in the previous subsection for the pair $A \subset p M p$. Take any increasing net $\left(p_{j}\right)_{j}$ of finite rank projections in $\mathbb{B}\left(L^{2}(M)\right)$. Under this setting, we can completely follow the proof of [27, Subsection 3.4]. Here we give a sketch of the proof for the reader's convenience.

By Lemmas 4.1.2 and 4.1.3, we have for any $S \in D_{0}$,

$$
\left|\Omega_{1}(\Theta(S))\right|=\limsup _{j}\left|\Omega_{1}\left(\Theta(S)\left(p_{j}^{\perp} \otimes 1\right)\right)\right| \leq \limsup _{j}\left\|\Theta(S)\left(p_{j}^{\perp} \otimes 1\right)\right\| \leq\|\Psi(S)\| .
$$

We can extend this inequality on $D$ (up to a scalar multiple) by condition (iv) of $\Omega_{1}$ and the $\mathrm{W}^{*} \mathrm{CBAP}$ of $M$ (use Lemma 2.3.1 if necessary). Then a positive functional $\Omega_{2}$ on $C^{*}\{\Psi(D)\}$ is defined by $\Omega_{2}(\Psi(X)):=\Omega_{1}(\Theta(X))$. This is a state since $\Omega_{2}(1)=1$. Take a Hahn-Banach extension of $\Omega_{2}$ on $\mathbb{B}\left(L^{2}(M) \otimes L^{2}(M) \otimes L^{2}(P)\right)$. Thanks to conditions (iv) and (vi) of $\Omega_{1}$, the restriction of this extended state on $p \mathbb{B}\left(L^{2}(M)\right) p \otimes \mathbb{C} 1_{M} \otimes \mathbb{C} 1_{P}$ is a $P$-central state which restricts $\operatorname{Tr}(p \cdot p)$ on $p M p$. Hence $P$ is injective.

4.3. Proof of Theorem B. We next prove Theorem $\mathrm{B}$ with a very similar argument. We actually prove the following statement.

Theorem 4.3.1. Let $M$ be a von Neumann algebra with separable predual and $\phi$ a faithful normal state on $M$. Let $N \subset M$ be a diffuse non-injective von Neumann subalgebra with a faithful normal conditional expectation $E_{N}$ which preserves $\phi$. If $(M, \phi)$ satisfies condition $(\mathrm{AOC})^{+}$and has the $W^{*} C B A P$, then $N$ has no $\phi$-Cartan subalgebras. 
Proof. Suppose by contradiction that $N$ has a $\phi$-Cartan subalgebra $B$ with a $\phi$ preserving conditional expectation $E_{B}$. Taking crossed products by $\mathbb{R}$ with the modular action of $\phi$, we have inclusions $B \otimes L \mathbb{R} \subset N \rtimes \mathbb{R}:=\mathcal{N} \subset M \rtimes \mathbb{R}:=\mathcal{M}$. Denote by $\operatorname{Tr}$ the canonical trace on $\mathcal{M}$. We can find a non-zero projection $p$ in $L \mathbb{R} \subset \mathcal{M}$ with $\operatorname{Tr}(p)<\infty$ such that $p \mathcal{N} p$ is still non-injective. Write $A:=B \otimes p L \mathbb{R} p$ and $P:=\mathcal{N}_{p \mathcal{M} p}(A)^{\prime \prime}$. Note that $P$ is non-injective since it contains the non-injective subalgebra $\mathcal{N}_{p \mathcal{N} p}(A)^{\prime \prime}=p \mathcal{N} p$ (see Subsection 2.1). We will apply almost the same argument as that in Theorem $\mathrm{A}$ to the inclusion $A \subset p \mathcal{M} p$ and will get injectivity of $P$, which means a contradiction.

Let $\left(\xi_{i}\right)_{i}, \Omega_{1} D, \Psi$, and $\Theta$ be as before for the pair $A \subset p \mathcal{M} p$ ( $\mathcal{M}$ has the $\mathrm{W}^{*} \mathrm{CBAP} ;$ see [1, 4.10]). We define $D_{0}:=M_{0} \rtimes_{r} \mathbb{R} \odot\left(M_{0} \rtimes_{r} \mathbb{R}\right)^{\text {op }} \odot P^{\mathrm{op}} \odot P$, where $M_{0}$ is a $\sigma$-weakly dense $C^{*}$-subalgebra of $M$ as in the definition of condition $(\mathrm{AOC})^{+}$. As mentioned in the observation below Theorem 4.1.1, we can choose $\xi_{i}$ as vectors in $L^{2}(\mathcal{M}, \tilde{\phi}) \otimes L^{2}(P)$. Recall $L^{2}(\mathcal{M}, \tilde{\phi})=L^{2}(M) \otimes L^{2}(\mathbb{R})$. In the setting, by a similar argument to that in Lemmas 4.1.2 and 4.1.3, we can prove the following statements:

- $\Omega_{1}\left(x \otimes 1_{L \mathbb{R}} \otimes 1_{P}\right)=0$ for any $x \in \mathbb{K}\left(L^{2}(M)\right)$;

- for any range finite projections $p_{j}$ in $\mathbb{B}\left(L^{2}(M)\right)$ with $p_{j} \rightarrow 1$ strongly, we have $\lim \sup _{j}\left\|\Theta(S)\left(p_{j}^{\perp} \otimes 1_{L \mathbb{R}} \otimes 1_{P}\right)\right\| \leq\|\Psi(S)\|$ for any $S \in D_{0}$.

Here we used diffuseness of $B$ (not of $A$ ) and condition $(\mathrm{AOC})^{+}$of $M$. Now we can completely follow the proof of Theorem $\mathrm{A}$ to get injectivity of $P$.

\section{Semisolidity of CONTINuOUs CORES}

In this section, we prove Theorem C. Our proof is a variant of the proof of [21, Theorem 4.6] and is very similar to that of [18, Theorem 5.3.3].

5.1. Proof of Theorem [C. For simplicity, we write the core of $M$ as $\mathcal{M}:=$ $M \rtimes_{\sigma^{\phi}} \mathbb{R}$. We use $L^{2}(\mathcal{M}, \tilde{\phi})=L^{2}(M) \otimes L^{2}(\mathbb{R})$ as a representation (although $\tilde{\phi}$ may not be a trace). Let $N$ be a type $\mathrm{II}_{1}$ subalgebra of $p \mathcal{M} p$. Since $N$ contains a copy of the AFD $\mathrm{II}_{1}$ factor, we may assume that $N$ itself is the AFD $\mathrm{II}_{1}$ factor. Let $N_{n} \subset N(n \in \mathbb{N})$ be an increasing finite dimensional unital $C^{*}$-subalgebra whose union is dense in $N$. We define a conditional expectation from $\mathbb{B}\left(L^{2}(\mathcal{M})\right)$ onto $N^{\prime} \cap p \mathbb{B}\left(L^{2}(\mathcal{M})\right) p=N^{\prime} p$ by

$$
\Psi_{N}(x):=\operatorname{Lim}_{n} \int_{\mathcal{U}\left(N_{n}\right)} u x u^{*} d u,
$$

where $d u$ is the normalized Haar measure on $\mathcal{U}\left(N_{n}\right)$ and Lim is taken by a fixed ultrafilter. Then $\Psi_{N}$ satisfies a properness condition:

$$
\Psi_{N}(x) \in \overline{\mathrm{co}}^{w}\left\{u x u^{*} \mid u \in \mathcal{U}(N)\right\} \quad\left(x \in \mathbb{B}\left(L^{2}(\mathcal{M})\right)\right) .
$$

We only prove that $\mathbb{K}\left(L^{2}(M)\right) \otimes \mathbb{B}\left(L^{2}(\mathbb{R})\right) \subset \operatorname{ker} \Psi_{N}$, and the theorem follows in the same manner as that in [21, Theorem 4.6] or [18, Theorem 5.3.3].

To see this, it suffices to show that $\Psi_{N}(x \otimes 1)=0$ for $x=J \hat{a} \otimes J \hat{b} \in \mathbb{K}\left(L^{2}(M)\right)$, where $a, b \in M$ and we used the Hilbert-Schmidt correspondence $(\xi \otimes \eta) \zeta:=\langle\zeta, \eta\rangle \xi$. Then since

$$
\Psi_{N}((J \hat{a} \otimes J \hat{b}) \otimes 1)=(J a J \otimes 1) \Psi_{N}((\hat{1} \otimes \hat{1}) \otimes 1)\left(J b^{*} J \otimes 1\right),
$$

we may assume $a=b=1$. Write $e:=\hat{1} \otimes \hat{1}$, which is the orthogonal projection onto $\mathbb{C} \hat{1}$. Let $q$ be any projection in $L \mathbb{R}$ with $\operatorname{Tr}(q)<\infty$. Put $\tilde{q}:=\tilde{J} q \tilde{J} \in \mathcal{M}^{\prime} \subset$ 
$N^{\prime}, \widetilde{N}:=N \tilde{q}$ and $\Psi_{\widetilde{N}}(x):=\tilde{q} \Psi_{N}(x) \tilde{q}$ for $x \in \mathbb{B}\left(L^{2}(\mathcal{M})\right)$. We actually prove $\Psi_{\widetilde{N}}(e \otimes 1)=0$ (this means $\Psi_{N}(e \otimes 1)=0$ by the choice of $\left.q\right)$.

Since $\Psi_{N}$ is proper, $\Psi_{N}(e \otimes 1)$ commutes with $\Delta_{\phi}^{i t} \otimes \rho_{t}$ and so it is contained in $p N^{\prime} \cap \rho(L \mathbb{R})^{\prime}$, where $\rho\left(\lambda_{t}\right):=\Delta_{\phi}^{i t} \otimes \rho_{t}$. Hence $\Psi_{\widetilde{N}}(e \otimes 1)$ is contained in $\tilde{q}\left(p N^{\prime} \cap \rho(L \mathbb{R})^{\prime}\right) \tilde{q} \subset N^{\prime} \cap \rho(q L \mathbb{R} q)^{\prime}$. Let $r$ be any spectral projection of $\Psi_{\widetilde{N}}(e \otimes 1)$ which corresponds to the interval $\left[\epsilon,\left\|\Psi_{\widetilde{N}}(e \otimes 1)\right\|\right]$ for any small $\epsilon>0$. Then since $r$ is also contained in $N^{\prime} \cap \rho(q L \mathbb{R} q)^{\prime}$ and $r \leq p \tilde{q}, r L^{2}(\mathcal{M})$ has a natural $N-q L \mathbb{R} q$ submodule structure of $p \tilde{q} L^{2}(\mathcal{M})\left(\simeq p \tilde{q} L^{2}(f \mathcal{M} f)\right.$, where $\left.f:=p \vee q\right)$. Since we know $N \swarrow_{f \mathcal{M} f} L \mathbb{R} q$ (because $N$ is of type $\mathrm{II}_{1}$ and $L \mathbb{R}$ is of type I), by Theorem 2.2.1 and the comment below it, the dimension of $r L^{2}(\mathcal{M})$ with respect to $\left(L \mathbb{R} q, \operatorname{Tr}_{L \mathbb{R} q}\right)$ is zero or infinite, where $\operatorname{Tr}_{L \mathbb{R} q}:=\operatorname{Tr}(\cdot) / \operatorname{Tr}(q)$ for the canonical trace $\operatorname{Tr}$ on $\mathcal{M}$.

Let $W$ be the unitary on $L^{2}(\mathcal{M})=L^{2}(M) \otimes L^{2}(\mathbb{R})$ given by $(W \xi)(t):=\Delta_{\phi}^{i t} \xi(t)$. Then easy calculations show that

$$
W\left(1 \otimes \lambda_{t}\right) W^{*}=\Delta^{i t} \otimes \lambda_{t}, \quad W\left(\Delta^{i t} \otimes \rho_{t}\right) W^{*}=1 \otimes \rho_{t},
$$

and hence we have

$$
\rho(q L \mathbb{R} q)^{\prime} \cap \mathbb{B}\left(L^{2}(\mathcal{M}) q\right)=\tilde{q} \rho(L \mathbb{R})^{\prime} \tilde{q}=W^{*}\left(\mathbb{B}\left(L^{2}(M)\right) \otimes \bar{q} L \mathbb{R}\right) W,
$$

where $\bar{q}:=J_{L \mathbb{R}} q J_{L \mathbb{R}}\left(\right.$ note $\left.1 \otimes \bar{q}=W \tilde{q} W^{*}\right)$. Then $\operatorname{dim}_{L \mathbb{R} q} r L^{2}(\mathcal{M})$ coincides with that of the right $L \mathbb{R} q$-module $W r W^{*}\left(L^{2}(M) \otimes L^{2}(\mathbb{R}) q\right)$, where the right action is given by $1 \otimes \rho_{t} \bar{q}(t \in \mathbb{R})$. From the fundamental theory of dimension, the dimension of $W r W^{*}\left(L^{2}(M) \otimes L^{2}(\mathbb{R}) q\right)$ is smaller than $\left(\operatorname{Tr}_{L^{2}(M)} \otimes \operatorname{Tr}\right)\left(W r W^{*}\right) / \operatorname{Tr}(q)$, and this is finite since

$$
\begin{aligned}
\left(\operatorname{Tr}_{L^{2}(M)} \otimes \operatorname{Tr}\right)\left(W r W^{*}\right) & \leq C \cdot\left(\operatorname{Tr}_{L^{2}(M)} \otimes \operatorname{Tr}\right)\left(W \Psi_{\widetilde{N}}(e \otimes 1) W^{*}\right) \\
& \leq C \cdot\left(\operatorname{Tr}_{L^{2}(M)} \otimes \operatorname{Tr}\right)\left(W \tilde{q}(e \otimes 1) \tilde{q} W^{*}\right) \\
& =C \cdot \operatorname{Tr}_{L^{2}(M)}(e) \operatorname{Tr}(\bar{q})<\infty
\end{aligned}
$$

where $C$ is a positive constant and we used properness of $\Psi_{N}$. Hence we have $r=0$ and $\Psi_{N}(e \otimes 1)=0$. Thus we proved the claim.

5.2. A remark on solidity and centralizer algebras. In the previous subsection, we proved semisolidity of continuous cores of some type $\mathrm{III}_{1}$ factors. This property itself has nothing to say about original type $\mathrm{III}_{1}$ factors at a first glance, but it has an interesting application once we get a stronger property, namely, solidity of the continuous cores. Indeed Houdayer gave the following observation [15, Subsection 3.3].

Let $M$ be a type $\mathrm{III}_{1}$ factor and assume that the continuous core $M \rtimes \mathbb{R}$ is solid as a $\mathrm{II}_{\infty}$ factor. Let $\phi$ be a faithful normal state on $M$ and $M_{\phi}$ be the centralizer of $\phi$ (see for example [30, Definition VIII.2.1]). Then by Takesaki's conditional expectation theorem [30, Theorem IX.4.2], there exists the unique $\phi$ preserving conditional expectation $E$ from $M$ onto $M_{\phi}$. Hence by the observation in Subsection 2.1, we have $M_{\phi} \otimes L \mathbb{R}=M_{\phi} \rtimes_{\sigma^{\phi}} \mathbb{R} \subset M \rtimes_{\sigma^{\phi}} \mathbb{R}$. Then for any Trfinite projection $p \in L \mathbb{R}, M_{\phi} \otimes \mathbb{C} p$ is injective since it is contained in the relative commutant of $L \mathbb{R} p$ and $L \mathbb{R}$ is diffuse. Thus the solidity of the continuous core forces all the centralizers (with respect to states) to be injective.

To apply this observation to our main objects, we next recall Connes' discrete decomposition of full type III factors [7, Section 4] (see also [10, Section 2]). 
Theorem 5.2.1. Let $M$ be a full type III factor with separable predual and $\phi$ a faithful normal state on $M$. Assume that $\phi$ is $\operatorname{Sd}(M)(=: \Gamma)$-almost periodic. Then there exists a decomposition $M \simeq\left(M \otimes \mathbb{B}\left(\ell^{2}(\Gamma)\right)\right)_{\phi \otimes \omega} \rtimes \Gamma$ with a faithful normal semifinite weight $\omega$ on $\mathbb{B}\left(\ell^{2}(\Gamma)\right)$. The algebra $\left(M \otimes \mathbb{B}\left(\ell^{2}(\Gamma)\right)\right)_{\phi \otimes \omega}$ is a $\mathrm{II}_{\infty}$ factor and is isomorphic to $M_{\phi} \otimes \mathbb{B}(H)$ for some separable Hilbert space $H$.

By the crossed product decomposition in the theorem, since $M$ is non-injective and $\Gamma$ is amenable, $\left(M \otimes \mathbb{B}\left(\ell^{2}(\Gamma)\right)\right)_{\phi \otimes \omega}$ is also non-injective. Hence we get noninjectivity of $M_{\phi}$.

We turn our attention to our main objects. Let $\mathbb{G}$ be a universal quantum group $A_{o}(F)$ for $F \in \operatorname{GL}(n, \mathbb{C})(n \geq 3)$ with $F \bar{F}= \pm 1$. Assume that $\|F\|^{2} \leq$ $\operatorname{Tr}_{n}\left(F F^{*}\right) / \sqrt{5}$, where $\operatorname{Tr}_{n}$ is the trace on $\mathbb{M}_{n}(\mathbb{C})$ with $\operatorname{Tr}_{n}(1)=n$. Then recall from [34, Theorem 7.1] that $L^{\infty}(\mathbb{G})$ then satisfies the following conditions:

- the algebra $L^{\infty}(\mathbb{G})$ is a full factor and the Haar state $h$ is almost periodic;

- the invariant $\operatorname{Sd}\left(L^{\infty}(\mathbb{G})\right)$ is the subgroup $\Gamma$ of $\mathbb{R}_{+}^{*}$ generated by eigenvalues of $Q \otimes Q^{-1}$, where $Q^{-1}:=F F^{*}$. In particular $L^{\infty}(\mathbb{G})$ is of type $\mathrm{II}_{1}$ if $F F^{*}=1$, of type $\operatorname{III}_{\lambda}(0<\lambda<1)$ if $\Gamma=\lambda^{\mathbb{Z}}$, and of type $\mathrm{III}_{1}$ in the other cases.

In this case, since the Haar state $h$ is $\operatorname{Sd}\left(L^{\infty}(\mathbb{G})\right)$-almost periodic, we have noninjectivity of $L^{\infty}(\mathbb{G})_{h}$ by the theorem above (in the $\mathrm{II}_{1}$ factor case $F F^{*}=1$, non-injectivity is trivial). Hence Houdayer's observation says that the continuous core of $L^{\infty}(\mathbb{G})$ is not solid. We summarize this result as follows.

Corollary 5.2.2. Let $\mathbb{G}$ be a universal quantum group $A_{o}(F)$ for $F \in \operatorname{GL}(n, \mathbb{C})$ $(n \geq 3)$ with $F \bar{F}= \pm 1$. Assume that $\|F\|^{2} \leq \operatorname{Tr}_{n}\left(F F^{*}\right) / \sqrt{5}$. Denote the continuous core of $L^{\infty}(\mathbb{G})$ by $\mathcal{M}$ and the canonical trace on $\mathcal{M}$ by $\operatorname{Tr}$. Then for any $\operatorname{Tr}$-finite projection $p$ in $\mathcal{M}$ with $p \mathcal{M} p$ non-injective, $p \mathcal{M} p$ is semisolid but never solid.

\section{ACKNOWLEDGEMENTS}

The author would like to thank Professors Yasuyuki Kawahigashi, who is his adviser, Eric Ricard, Reiji Tomatsu, Yoshimichi Ueda, and Makoto Yamashita for their valuable comments. In particular, he thanks his colleague Yuki Arano for fruitful conversations on compact quantum groups. The author was supported as a Research Fellow of the Japan Society for the Promotion of Science.

\section{REFERENCES}

[1] C. Anantharaman-Delaroche, Amenable correspondences and approximation properties for von Neumann algebras, Pacific J. Math. 171 (1995), no. 2, 309-341. MR.1372231(96m:46106)

[2] Teodor Banica, Le groupe quantique compact libre $\mathrm{U}(n)$ (French, with English summary), Comm. Math. Phys. 190 (1997), no. 1, 143-172, DOI 10.1007/s002200050237. MR.1484551 (99k:46095)

[3] Teodor Banica, Théorie des représentations du groupe quantique compact libre $\mathrm{O}(n)$ (French, with English and French summaries), C. R. Acad. Sci. Paris Sér. I Math. 322 (1996), no. 3, 241-244. MR 1378260 (97a:46108)

[4] Nathanial P. Brown and Narutaka Ozawa, $C^{*}$-algebras and finite-dimensional approximations, Graduate Studies in Mathematics, vol. 88, American Mathematical Society, Providence, RI, 2008. MR2391387 (2009h:46101)

[5] Michael Brannan, Approximation properties for free orthogonal and free unitary quantum groups, J. Reine Angew. Math. 672 (2012), 223-251. MR2995437 
[6] Man Duen Choi and Edward G. Effros, The completely positive lifting problem for $C^{*}$ algebras, Ann. of Math. (2) 104 (1976), no. 3, 585-609. MR0417795 (54 \#5843)

[7] A. Connes, Almost periodic states and factors of type $\mathrm{III}_{1}$, J. Functional Analysis 16 (1974), 415-445. MR0358374 (50 \#10840)

[8] Ionut Chifan and Thomas Sinclair, On the structural theory of $\Pi_{1}$ factors of negatively curved groups (English, with English and French summaries), Ann. Sci. Éc. Norm. Supér. (4) 46 (2013), no. 1, 1-33 (2013). MR 3087388

[9] Ionut Chifan, Thomas Sinclair, and Bogdan Udrea, On the structural theory of II $I_{1}$ factors of negatively curved groups, II: Actions by product groups, Adv. Math. 245 (2013), 208-236, DOI 10.1016/j.aim.2013.06.017. MR 3084428

[10] Kenneth Dykema, Crossed product decompositions of a purely infinite von Neumann algebra with faithful, almost periodic weight, Indiana Univ. Math. J. 44 (1995), no. 2, 433-450, DOI 10.1512/iumj.1995.44.1995. MR.1355406 (96k:46106)

[11] Junsheng Fang, Roger R. Smith, and Stuart White, Groupoid normalisers of tensor products: infinite von Neumann algebras, J. Operator Theory 69 (2013), no. 2, 545-570, DOI 10.7900/jot.2011mar05.1928. MR 3053355

[12] Jacob Feldman and Calvin C. Moore, Ergodic equivalence relations, cohomology, and von Neumann algebras. I, II, Trans. Amer. Math. Soc. 234 (1977), no. 2, 289-324; 325-359. MR0578656(58\#28261a) MR0578730 (58 \#28261b)

[13] Amaury Freslon, Examples of weakly amenable discrete quantum groups, J. Funct. Anal. 265 (2013), no. 9, 2164-2187, DOI 10.1016/j.jfa.2013.05.037. MR.3084500

[14] Mingchu Gao and Marius Junge, Examples of prime von Neumann algebras, Int. Math. Res. Not. IMRN 15 (2007), Art. ID rnm042, 34, DOI 10.1093/imrn/rnm042. MR.2348404 (2008g:46107)

[15] Cyril Houdayer, Structural results for free Araki-Woods factors and their continuous cores, J. Inst. Math. Jussieu 9 (2010), no. 4, 741-767, DOI 10.1017/S1474748010000058. MR2684260 (2011i:46069)

[16] Cyril Houdayer and Éric Ricard, Approximation properties and absence of Cartan subalgebra for free Araki-Woods factors, Adv. Math. 228 (2011), no. 2, 764-802, DOI 10.1016/j.aim.2011.06.010. MR.2822210 (2012f:46116)

[17] Cyril Houdayer and Stefaan Vaes, Type III factors with unique Cartan decomposition (English, with English and French summaries), J. Math. Pures Appl. (9) 100 (2013), no. 4, 564-590, DOI 10.1016/j.matpur.2013.01.013. MR3102166

[18] Yusuke Isono, Weak exactness for $C^{*}$-algebras and application to condition (AO), J. Funct. Anal. 264 (2013), no. 4, 964-998, DOI 10.1016/j.jfa.2012.10.021. MR3004955

[19] Kenley Jung, Strongly 1-bounded von Neumann algebras, Geom. Funct. Anal. 17 (2007), no. 4, 1180-1200, DOI 10.1007/s00039-007-0624-9. MR2373014(2009i:46115)

[20] Ann Maes and Alfons Van Daele, Notes on compact quantum groups, Nieuw Arch. Wisk. (4) 16 (1998), no. 1-2, 73-112. MR.1645264 (99g:46105)

[21] Narutaka Ozawa, A Kurosh-type theorem for type $\mathrm{II}_{1}$ factors, Int. Math. Res. Not. 2006, Art. ID 97560, 21 pp., DOI 10.1155/IMRN/2006/97560. MR2211141 (2006m:46078)

[22] Narutaka Ozawa, Examples of groups which are not weakly amenable, Kyoto J. Math. 52 (2012), no. 2, 333-344, DOI 10.1215/21562261-1550985. MR2914879

[23] Narutaka Ozawa, Solid von Neumann algebras, Acta Math. 192 (2004), no. 1, 111-117, DOI 10.1007/BF02441087. MR2079600(2005e:46115)

[24] Narutaka Ozawa and Sorin Popa, On a class of $\mathrm{II}_{1}$ factors with at most one Cartan subalgebra, Ann. of Math. (2) 172 (2010), no. 1, 713-749, DOI 10.4007/annals.2010.172.713. MR2680430 (2011j:46101)

[25] Sorin Popa, Strong rigidity of $\mathrm{II}_{1}$ factors arising from malleable actions of w-rigid groups. I, Invent. Math. 165 (2006), no. 2, 369-408, DOI 10.1007/s00222-006-0501-4. MR.2231961 (2007f:46058)

[26] Sorin Popa, On a class of type $\mathrm{II}_{1}$ factors with Betti numbers invariants, Ann. of Math. (2) 163 (2006), no. 3, 809-899, DOI 10.4007/annals.2006.163.809. MR2215135(2006k:46097)

[27] Sorin Popa and Stefaan Vaes, Unique Cartan decomposition for $\mathrm{II}_{1}$ factors arising from arbitrary actions of hyperbolic groups, J. Reine Angew. Math. 694 (2014), 215-239, DOI 10.1515/crelle-2012-0104. MR3259044 
[28] Dimitri Shlyakhtenko, Some estimates for non-microstates free entropy dimension with applications to q-semicircular families, Int. Math. Res. Not. 51 (2004), 2757-2772, DOI 10.1155/S1073792804140476. MR2130608(2006e:46074)

[29] Dimitri Shlyakhtenko, Prime type III factors, Proc. Natl. Acad. Sci. USA 97 (2000), no. 23, 12439-12441 (electronic), DOI 10.1073/pnas.220417397. MR.1791311 (2002c:46121)

[30] M. Takesaki, Theory of operator algebras. II, Encyclopaedia of Mathematical Sciences, vol. 125, Operator Algebras and Non-commutative Geometry, 6, Springer-Verlag, Berlin, 2003. MR.1943006 (2004g:46079)

[31] Reiji Tomatsu, A characterization of right coideals of quotient type and its application to classification of Poisson boundaries, Comm. Math. Phys. 275 (2007), no. 1, 271-296, DOI 10.1007/s00220-007-0267-6. MR2335776 (2008j:46058)

[32] Roland Vergnioux, Orientation of quantum Cayley trees and applications, J. Reine Angew. Math. 580 (2005), 101-138, DOI 10.1515/crll.2005.2005.580.101. MR2130588(2006f:46062)

[33] Stefaan Vaes and Nikolas Vander Vennet, Poisson boundary of the discrete quantum group $\widehat{A_{u}(F)}$, Compos. Math. 146 (2010), no. 4, 1073-1095, DOI 10.1112/S0010437X1000477X. MR:2660685 (2011m:46120)

[34] Stefaan Vaes and Roland Vergnioux, The boundary of universal discrete quantum groups, exactness, and factoriality, Duke Math. J. 140 (2007), no. 1, 35-84, DOI 10.1215/S00127094-07-14012-2. MR2355067 (2010a:46166)

[35] Alfons Van Daele and Shuzhou Wang, Universal quantum groups, Internat. J. Math. 7 (1996), no. 2, 255-263, DOI 10.1142/S0129167X96000153. MR.1382726 (97d:46090)

[36] D. Voiculescu, The analogues of entropy and of Fisher's information measure in free probability theory. III. The absence of Cartan subalgebras, Geom. Funct. Anal. 6 (1996), no. 1, 172-199, DOI 10.1007/BF02246772. MR1371236(96m:46119)

[37] S. L. Woronowicz, Compact quantum groups, Symétries quantiques (Les Houches, 1995), North-Holland, Amsterdam, 1998, pp. 845-884. MR1616348 (99m:46164)

Department of Mathematical Sciences, University of Tokyo, Komaba, Tokyo, 1538914, JAPAN

Current address: Research Institute for Mathematical Sciences, Kyoto University, Kyoto 6068502, Japan

E-mail address: isono@kurims.kyoto-u.ac.jp 